Infinite Dimensional Analysis, Quantum Probability and Related Topics

(C) World Scientific Publishing Company

\title{
ASYMPTOTICS OF STOCHASTIC 2D HYDRODYNAMICAL TYPE SYSTEMS IN UNBOUNDED DOMAINS
}

\author{
JUAN YANG \\ School of Science, Beijing University of posts and telecommunications, \\ No.10 Xitucheng Road, Haidian District, Beijing, 100875, The People's Republican of China \\ yangjuanyj6@gmail.com \\ JIANLIANG ZHAI \\ School of Mathematical Sciences, University of Science and Technology of China, \\ Key Laboratory of Wu Wen-Tsun Mathematics, Chinese Academy of Sciences, \\ No. 96 Jinzhai Road, Hefei, 230026, The People's Republican of China \\ zhaijl@ustc.edu.cn \\ Received (Day Month Year) \\ Revised (Day Month Year) \\ Published (Day Month Year) \\ Communicated by (JIANLIANG ZHAI)
}

\begin{abstract}
In this paper, we prove a central limit theorem and establish a moderate deviation principle for $2 \mathrm{D}$ stochastic hydrodynamical type systems with multiplicative noise in unbounded domains, which covers 2D Navier-Stokes equations, 2D MHD models and the 2D magnetic Bénard problem and also shell models of turbulence. The weak convergence method plays an important role in obtaining the moderate deviation principle.
\end{abstract}

Keywords: Stochastic hydrodynamical type systems; Central limit theorem; Moderate deviation principle.

AMS Subject Classification: 60H15, 60F05, 60F10

\section{Introduction}

Let $(\Omega, \mathcal{F}, \mathbb{P})$ be a probability space with an increasing family $\left\{\mathcal{F}_{t}\right\}_{0 \leq t \leq T}$ of the sub$\sigma$-fields of $\mathcal{F}$ satisfying the usual conditions. Consider abstract stochastic evolution equation of the following form

$$
\partial_{t} u+\mathcal{A} u+\mathcal{B}(u, u)+\mathcal{R}(u)=\sigma(t, u) \dot{W}(t),
$$

where $W(\cdot)$ is a Wiener process. This abstract nonlinear form covers a host class of 2D hydrodynamical type systems including the following models. (1) When $\mathcal{R}=0$, it is 2D Navier-Stokes equations. (2) 2D magneto-hydrodynamic equations are a combination of the Navier-Stokes equations of fluid dynamics and Maxwell's equations of electromagnetism. Magneto-hydrodynamics is the study of the magnetic properties of electrically conducting fluids like plasmas, liquid metals, salt water or 
electrolytes. (3) The Bénard problem is a viscous fluid, in a rectangular container $\mathrm{D}$, which is heated from below and the top surface is taken at constant temperature. 2D Boussinesq model for the Bénard convection is the Navier-Stokes equations coupled with equations for temperature, i.e. heat equations. (4) 2D magnetic Bénard problem is the Boussinesq model coupled with magnetic field. (5) 3D Leray $\alpha$-model is quite similar to the Lagrangian averaged Navier-Stokes $\alpha$-model of turbulence. The computational overhead connected with the Leray $\alpha$-model was lower than that of dynamic models, like the Lagrangian averaged Navier-Stokes $\alpha$-model and other sub-grid scale models of turbulence. (6) Shell models of turbulence are useful phenomenological models that retain certain features of the Navier-Stokes equations. These models are the Navier-Stokes equations written in Fourier space containing only local interaction between the modes. Their main computational advantage is the parameterization of the fluctuation of a turbulent field in each octave of wave numbers (called shells) by few representative variables. We refer to 7 and the references therein for examples and more details.

Let $u^{\varepsilon}$ denote the solution of the following equation

$$
d u^{\varepsilon}(t)+A u^{\varepsilon}(t) d t+B\left(u^{\varepsilon}(t)\right) d t+\tilde{R}\left(t, u^{\varepsilon}(t)\right) d t=\sqrt{\varepsilon} \sigma\left(t, u^{\varepsilon}(t)\right) d W(t),
$$

with the initial condition $u^{\varepsilon}(0)=\xi$ for some fixed point $\xi$ in $H$. As the parameter $\varepsilon$ tends to zero, the solution $u^{\varepsilon}$ of (1.2) will tend to the solution $u^{0}$ of the following deterministic equation

$$
d u^{0}(t)+A u^{0}(t) d t+B\left(u^{0}(t)\right) d t+\tilde{R}\left(t, u^{0}(t)\right) d t=0, \quad \text { with } u^{0}(0)=\xi \in H .
$$

The aim of this paper is to study deviations of $u^{\epsilon}$ from $u^{0}$ as $\epsilon \rightarrow 0$. That is, the asymptotic behavior of the trajectory

$$
\frac{1}{\sqrt{\epsilon} \lambda(\varepsilon)}\left(u^{\epsilon}-u^{0}\right)(t), \quad t \in[0, T] .
$$

When $\lambda(\varepsilon)=1$, it is related to central limit theorem (CLT for short). And when $\lambda(\varepsilon)$ is the deviation scale verifies

$$
\lambda(\varepsilon) \rightarrow+\infty, \quad \sqrt{\varepsilon} \lambda(\varepsilon) \rightarrow 0, \text { as } \varepsilon \rightarrow 0,
$$

it provides so-called moderate deviation principle (MDP for short, cf. 8 ). Throughout the paper, we assume that (1.4) is in place.

Moderate deviation is an intermediate estimation between the large deviation with scale $\lambda(\varepsilon)=1 / \sqrt{\varepsilon}$ and the CLT. Like the large deviations, the moderate deviation theory stems from inferential theory of statistics. In recent years, there is an increasing interest on the study of MDP. For independent and identically distributed random sequences, Chen 4, 5, 6] and Ledoux 16 found the necessary and sufficient conditions for MDP. For Markov chain processes, moderate deviation was discussed by Djellout and Guillin 9, Gao 14, 15, and Wu 20, 21, Baier and Freidlin 1 and Guillin 13 considered models with averaging. Moderate deviations for mean field interacting particle models was worked by Douc, Guillin and Najim 
11 and Del Moral, Hu and Wu 12, Wang and Zhang 17, Wang, Zhai and Zhang 19 discussed moderate deviations for stochastic reaction-diffusion equation and 2D stochastic Navier-Stokes equations, respectively. Recently, there are several works on moderate deviations for SPDEs with jump, see 3, 10, 18.

In this paper, we prove a central limit theorem and establish a moderate deviation principle for the class of abstract nonlinear stochastic models of the form (1.2) with multiplicative noise in unbounded domains, which cover a wide class of mathematical coupled models from fluid dynamics mentioned in first sentence. There are two main difficulties. One is caused by the bilinear term in hydrodynamical systems, we have to deal with the moment of the norm delicately. Another one is that, since we do not assume the compactness of embeddings in the corresponding Gelfand triple $V \subset H \subset V^{\prime}$, this allows us to cover the important class of hydrodynamical models in unbounded domains. The payoff is that we have to impose some more time regularity assumption on the diffusion coefficient (see (4.5)), much of the problem is caused by this. This is the main difference from 19. The technique introduced by 7 will be used in our paper.

The organization of this paper is as follows. In Section 2, we shall introduce stochastic 2D hydrodynamical type systems. We prove the CLT in Section 3. Section 4 is devoted to show the MDP.

Throughout this paper, $C$ is a positive constant independent of $\varepsilon$ and its value may be different from line to line.

\section{Description of the Model}

To formulate the stochastic evolution equation (1.2), we introduce the following standard spaces: let $(H,|\cdot|,(\cdot, \cdot))$ denote a separable Hilbert space, and $A$ be an selfadjoint positive linear operator on $\mathrm{H}$. Set $V=\operatorname{Dom}\left(A^{\frac{1}{2}}\right)$ with norm $\|v\|:=\left|A^{\frac{1}{2}} v\right|$, where $v \in V$. Let $V^{\prime}$ be the dual of $V$ and $(u, v)$ denote the duality between $u \in V$ and $v \in V^{\prime}$. Identifying $H$ with its dual $H^{\prime}$, we have the dense, continuous embedding $V \hookrightarrow H \cong H^{\prime} \hookrightarrow V^{\prime}$.

Consider the following stochastic equation:

$$
d u(t)+A u(t)+B(u(t))+\tilde{R}(t, u(t))=\sigma(t, u(t)) d W(t),
$$

where $B(u):=B(u, u)$.

We assume that the mapping $B: V \times V \rightarrow V^{\prime}$ satisfies the following antisymmetry and bounded conditions:

(C1-1) $B: V \times V \rightarrow V^{\prime}$ is a bilinear continuous mapping.

(C1-2) For $u_{1}, u_{2}, u_{3} \in V$,

$$
\left(B\left(u_{1}, u_{2}\right), u_{3}\right)=-\left(B\left(u_{1}, u_{3}\right), u_{2}\right) .
$$

(C1-3) There exists a Banach interpolation space $\mathcal{H}$ possessing the properties: 
(i) $V \hookrightarrow \mathcal{H} \hookrightarrow V^{\prime}$;

(ii) there exists a constant $a_{0}>0$ such that for any $v \in V$,

$$
\|v\|_{\mathcal{H}}^{2} \leq a_{0}|v|\|v\|
$$

(iii) for every $\eta>0$ there exists $C_{\eta}>0$ such that for $u_{1}, u_{2}, u_{3} \in V$,

$$
\left|\left(B\left(u_{1}, u_{2}\right), u_{3}\right)\right| \leq \eta\left\|u_{3}\right\|^{2}+C_{\eta}\left\|u_{1}\right\|_{\mathcal{H}}^{2}\left\|u_{2}\right\|_{\mathcal{H}}^{2} .
$$

It is evident that (2.4) is equivalent to the following two inequalities (see Remark 2.1 in (7),

$$
\begin{gathered}
\left|\left(B\left(u_{1}, u_{2}\right), u_{3}\right)\right| \leq C_{1}\left\|u_{3}\right\|^{2}+C_{2}\left\|u_{1}\right\|_{\mathcal{H}}^{2}\left\|u_{2}\right\|_{\mathcal{H}}^{2} ; \\
\left|\left(B\left(u_{1}, u_{2}\right), u_{3}\right)\right| \leq C\left\|u_{1}\right\|_{\mathcal{H}}^{2}\left\|u_{2}\right\|\left\|u_{3}\right\|_{\mathcal{H}}^{2},
\end{gathered}
$$

where $C_{1}, C_{2}, C$ are some positive constants.

In view of (2.2), (2.3) and (2.6), it yields

$$
\left|\left(B\left(u_{1}, u_{1}\right), u_{2}\right)\right| \leq \eta\left\|u_{1}\right\|^{2}+C_{\eta}\left|u_{1}\right|^{2}\left\|u_{2}\right\|_{\mathcal{H}}^{4} .
$$

And then,

$\left|\left(B\left(u_{1}\right)-B\left(u_{2}\right), u_{1}-u_{2}\right)\right|=\left|\left(B\left(u_{1}-u_{2}\right), u_{2}\right)\right| \leq \eta|| u_{1}-u_{2}\left\|^{2}+C_{\eta}\left|u_{1}-u_{2}\right|^{2}\right\| u_{2} \|_{\mathcal{H}}^{4}$.

The covariance operator $Q$ of the Wiener process $W(\cdot)$ is a positive symmetric, trace class operator on $H$. Let $H_{0}=Q^{1 / 2} H$. Then $H_{0}$ is a Hilbert space with the inner product

$$
\langle u, v\rangle_{0}=\left(Q^{-1 / 2} u, Q^{-1 / 2} v\right) \quad \forall u, v \in H_{0} .
$$

Let $|\cdot|_{0}$ denote the norm in $H_{0}$. Clearly, the embedding of $H_{0}$ in $H$ is HilbertSchmidt, since $Q$ is a trace class operator. Let $L_{Q}\left(H_{0} ; H\right)$ denote the space of linear operators $S$ such that $S Q^{1 / 2}$ is a Hilbert-Schmidt operator from $H$ to $H$. Define the norm on the space $L_{Q}\left(H_{0} ; H\right)$ by $|S|_{L_{Q}}=\sqrt{\operatorname{tr}\left(S Q S^{*}\right)}$. Set $L_{\left(H_{0}, H\right)}$ the space of all bounded linear operators from $H_{0}$ into $H$, and denote $|\cdot|_{L_{\left(H_{0}, H\right)}}$ its norm.

The noise coefficient $\sigma \in C\left([0, T] \times V ; L_{Q}\left(H_{0} ; H\right)\right)$ satisfies the following hypothesis, for all $t \in[0, T], u, v \in V$,

$(\mathrm{C} 2-1)|\sigma(t, u)|_{L_{Q}}^{2} \leq K_{0}+K_{1}|u|^{2}$, for some $K_{0}, K_{1}>0$.

$(\mathrm{C} 2-2)|\sigma(t, u)-\sigma(t, v)|_{L_{Q}}^{2} \leq L_{1}|u-v|^{2}$, for some $L_{1}>0$.

We introduce another coefficient $\tilde{R} \in C([0, T] \times H ; H)$ which satisfy, for some $R_{0}, R_{1}>0$,

(C3) $|\tilde{R}(t, 0)| \leq R_{0},|\tilde{R}(t, u)-\tilde{R}(t, v)| \leq R_{1}|u-v|, \forall t \in[0, T], u, v \in H$.

From the Theorem 2.4 in 7 , the following lemma holds.

Lemma 2.1 Assume that (C1-1)-(C1-3), (C2-1)-(C2-2) and (C3) hold. There exists a constant $\varepsilon_{0}>0$ such that for any $0<\varepsilon \leq \varepsilon_{0}$, (1.2) has a unique weak solution 
$u^{\varepsilon}$ in $L^{2}(\Omega ; C([0, T] ; H)) \cap L^{2}(\Omega \times[0, T] ; V)$. Furthermore, for some constant $C$ independent of $\varepsilon$,

$\mathbb{E}\left(\sup _{0 \leq t \leq T}\left|u^{\varepsilon}(t)\right|^{4}+\int_{0}^{T}\left\|u^{\varepsilon}(s)\right\|^{2} d s+\int_{0}^{T}\left|u^{\varepsilon}(s)\right|^{2} \cdot\left\|u^{\varepsilon}(s)\right\|^{2} d s+\int_{0}^{T}\left\|u^{\varepsilon}(s)\right\|_{\mathcal{H}}^{4} d s\right) \leq C\left(1+|\xi|^{4}\right) ;$

and particulary,

$\sup _{0 \leq t \leq T}\left|u^{0}(t)\right|^{4}+\int_{0}^{T}\left\|u^{0}(s)\right\|^{2} d s+\int_{0}^{T}\left|u^{0}(s)\right|^{2} \cdot\left\|u^{0}(s)\right\|^{2} d s+\int_{0}^{T}\left\|u^{0}(s)\right\|_{\mathcal{H}}^{4} d s \leq C\left(1+|\xi|^{4}\right)$,

here $u^{0}$ is the solution of (1.3).

\section{Central Limit Theorem}

In this section, we will establish the CLT.

The following result is concerned with the convergence of $u^{\varepsilon}$ as $\varepsilon \rightarrow 0$, and can be obtained similarly as Proposition 3.1 in 19 . The proof is omitted.

Proposition 3.1 Under the conditions (C1-1)-(C1-3), (C2-1)-(C2-2) and (C3), there exists a constant $\varepsilon_{0}>0$ such that, for all $0<\varepsilon \leq \varepsilon_{0}$,

$$
\mathbb{E}\left(\sup _{0 \leq t \leq T}\left|u^{\varepsilon}(t)-u^{0}(t)\right|^{2}+\int_{0}^{T}\left\|u^{\varepsilon}(s)-u^{0}(s)\right\|^{2} d s\right) \leq \varepsilon c_{T, K_{0}, K_{1}} .
$$

Let $V^{0}$ be the solution of the following SPDE:

$d V^{0}(t)+A V^{0}(t) d t+B\left(V^{0}(t), u^{0}(t)\right)+B\left(u^{0}(t), V^{0}(t)\right) d t+\tilde{R}^{\prime}\left(t, u^{0}(t)\right) V^{0}(t) d t=\sigma\left(t, u^{0}(t)\right) d W(t)$,

with initial value $V^{0}(0)=0$, where $\tilde{R}^{\prime}$ given in $(\mathrm{C} 4)$. For the existence and uniqueness of the solution for (3.2), we need the following additional assumption about $\tilde{R}^{\prime}$.

(C4) $\tilde{R}^{\prime}:[0, T] \times H \rightarrow L(H)$ is Fréchet derivative of $\tilde{R}$ w.r.t. the second variable and $\tilde{R}^{\prime}$ is continuous such that $\left|\tilde{R}^{\prime}(t, u)\right|_{L(H)} \leq \tilde{R}_{0}^{\prime}|u|+\tilde{R}_{1}^{\prime}$ for some positive $\tilde{R}_{0}^{\prime}$ and $\tilde{R}_{1}^{\prime}$.

Similarly as the proof in Theorem 2.4 in 7 , the following lemma holds.

Lemma 3.1 Assume that (C1-1)-(C1-3), (C2-1)-(C2-2) and (C4) hold. (3.2) has a unique weak solution $V^{0}$ in $L^{2}(\Omega ; C([0, T] ; H)) \cap L^{2}(\Omega \times[0, T] ; V)$. Furthermore, the solution has the following estimate, for some constant $C>0$,

$\mathbb{E}\left(\sup _{0 \leq t \leq T}\left|V^{0}(t)\right|^{4}+\int_{0}^{T}\left\|V^{0}(s)\right\|^{2} d s+\int_{0}^{T}\left|V^{0}(s)\right|^{2}\left\|V^{0}(s)\right\|^{2} d s+\int_{0}^{T}\left\|V^{0}(s)\right\|_{\mathcal{H}}^{4} d s\right) \leq C\left(1+|\xi|^{4}\right) ;$ 
Our first main result is the CLT. We add the following assumption on $\tilde{R}^{\prime}$.

(C5) There exists $C>0$ such that, for every $u_{1}, u_{2} \in H$,

$$
\left|\tilde{R}^{\prime}\left(t, u_{1}\right)-\tilde{R}^{\prime}\left(t, u_{2}\right)\right|_{L(H)} \leq C\left|u_{1}-u_{2}\right| .
$$

Theorem 3.2 (Central Limit Theorem) Under the conditions (C1-1)-(C1-3), (C2-1)-(C2-2) and (C3)-(C5), $\left(u^{\varepsilon}-u^{0}\right) / \sqrt{\varepsilon}$ converges to $V^{0}$ in the space $C([0, T] ; H) \cap L^{2}([0, T] ; V)$ in probability, that is, as $\varepsilon \rightarrow 0$,

$\sup _{0 \leq t \leq T}\left|\frac{u^{\varepsilon}(t)-u^{0}(t)}{\sqrt{\varepsilon}}-V^{0}(t)\right|^{2}+\int_{0}^{T}\left\|\frac{u^{\varepsilon}(s)-u^{0}(s)}{\sqrt{\varepsilon}}-V^{0}(s)\right\|^{2} d s \rightarrow 0$, in probability.

Remark 3.1 Because of the property of $\tilde{R}^{\prime}$, we can only obtain 3.4, which is weaker than Theorem 3.2 in 19, i.e.

$$
\lim _{\varepsilon \rightarrow 0} \mathbb{E}\left(\sup _{0 \leq t \leq T}\left|\frac{u^{\varepsilon}(t)-u^{0}(t)}{\sqrt{\varepsilon}}-V^{0}(t)\right|^{2}+\int_{0}^{T}\left\|\frac{u^{\varepsilon}(s)-u^{0}(s)}{\sqrt{\varepsilon}}-V^{0}(s)\right\|^{2} d s\right)=0 .
$$

Proof. Let $V^{\varepsilon}(t):=\left(u^{\varepsilon}(t)-u^{0}(t)\right) / \sqrt{\varepsilon}$. Then $V^{\varepsilon}(0)=0$,

$$
\begin{aligned}
& d V^{\varepsilon}(t)+A V^{\varepsilon}(t) d t+B\left(V^{\varepsilon}(t), u^{\varepsilon}(t)\right) d t+B\left(u^{0}(t), V^{\varepsilon}(t)\right) d t \\
& +\frac{1}{\sqrt{\varepsilon}}\left[\tilde{R}\left(t, u^{\varepsilon}(t)\right)-\tilde{R}\left(t, u^{0}(t)\right)\right]=\sigma\left(t, u^{\varepsilon}(t)\right) d W(t),
\end{aligned}
$$

and

$$
\begin{aligned}
& d\left(V^{\varepsilon}(t)-V^{0}(t)\right)+A\left(V^{\varepsilon}(t)-V^{0}(t)\right) d t \\
& +B\left(V^{\varepsilon}(t)-V^{0}(t), u^{0}(t)\right) d t+B\left(V^{\varepsilon}(t), u^{\varepsilon}(t)-u^{0}(t)\right) d t+B\left(u^{0}(t), V^{\varepsilon}(t)-V^{0}(t)\right) d t \\
& +\frac{1}{\sqrt{\varepsilon}}\left[\tilde{R}\left(t, u^{\varepsilon}(t)\right)-\tilde{R}\left(t, u^{0}(t)\right)\right] d t-\tilde{R}^{\prime}\left(t, u^{0}(t)\right) V^{0}(t) d t \\
= & \left.\sigma\left(t, u^{\varepsilon}(t)\right)-\sigma\left(t, u^{0}(t)\right)\right] d W(t) .
\end{aligned}
$$

By Itô's formula for $\left|V^{\varepsilon}(t)-V^{0}(t)\right|^{2}$ and (2.2), we have

$$
\begin{aligned}
d \mid & V^{\varepsilon}(t)-\left.V^{0}(t)\right|^{2}+2\left\|V^{\varepsilon}(t)-V^{0}(t)\right\|^{2} d t \\
= & -2\left(B\left(V^{\varepsilon}(t)-V^{0}(t), u^{0}(t)\right), V^{\varepsilon}(t)-V^{0}(t)\right) d t \\
& -2\left(B\left(V^{\varepsilon}(t), u^{\varepsilon}(t)-u^{0}(t)\right), V^{\varepsilon}(t)-V^{0}(t)\right) d t \\
& -2\left(\frac{1}{\sqrt{\varepsilon}}\left[\tilde{R}\left(t, u^{\varepsilon}(t)\right)-\tilde{R}\left(t, u^{0}(t)\right)\right]-\tilde{R}^{\prime}\left(t, u^{0}(t)\right) V^{0}(t), V^{\varepsilon}(t)-V^{0}(t)\right) d t \\
& +2\left(\left[\sigma\left(t, u^{\varepsilon}(t)\right)-\sigma\left(t, u^{0}(t)\right)\right] d W(t), V^{\varepsilon}(t)-V^{0}(t)\right) \\
& +\left|\sigma\left(t, u^{\varepsilon}(t)\right)-\sigma\left(t, u^{0}(t)\right)\right|_{L_{Q}}^{2} d t .
\end{aligned}
$$


Defining $\tau_{N}=\inf \left\{t:\left|V^{\varepsilon}(t)-V^{0}(t)\right|^{2}+\int_{0}^{t}\left\|V^{\varepsilon}(s)-V^{0}(s)\right\|^{2} d s>N\right\}$, we have

$$
\begin{aligned}
& \quad\left|V^{\varepsilon}\left(t \wedge \tau_{N}\right)-V^{0}\left(t \wedge \tau_{N}\right)\right|^{2}+2 \int_{0}^{t \wedge \tau_{N}}\left\|V^{\varepsilon}(s)-V^{0}(s)\right\|^{2} d s \\
& \leq 2 \int_{0}^{t \wedge \tau_{N}}\left|\left(B\left(V^{\varepsilon}(s)-V^{0}(s), u^{0}(s)\right), V^{\varepsilon}(s)-V^{0}(s)\right)\right| d s \\
& \quad+2 \int_{0}^{t \wedge \tau_{N}}\left|\left(B\left(V^{\varepsilon}(s), u^{\varepsilon}(s)-u^{0}(s)\right), V^{\varepsilon}(s)-V^{0}(s)\right)\right| d s \\
& \quad+2 \int_{0}^{t \wedge \tau_{N}}\left|\left(\frac{1}{\sqrt{\varepsilon}}\left[\tilde{R}\left(s, u^{\varepsilon}(s)\right)-\tilde{R}\left(s, u^{0}(s)\right)\right]-\tilde{R}^{\prime}\left(s, u^{0}(s)\right) V^{0}(s), V^{\varepsilon}(s)-V^{0}(s)\right)\right| d s \\
& \quad+2\left|\int_{0}^{t \wedge \tau_{N}}\left(\left[\sigma\left(s, u^{\varepsilon}(s)\right)-\sigma\left(s, u^{0}(s)\right)\right] d W(s), V^{\varepsilon}(s)-V^{0}(s)\right)\right| \\
& \quad+\int_{0}^{t \wedge \tau_{N}}\left|\sigma\left(s, u^{\varepsilon}(s)\right)-\sigma\left(s, u^{0}(s)\right)\right|_{L_{Q}}^{2} d s \\
& =: \sum_{k=1}^{5} I_{k} .
\end{aligned}
$$

Because of (2.2) and (2.7), we have

$$
I_{1}(t) \leq 2 \eta \int_{0}^{t \wedge \tau_{N}}\left\|V^{\varepsilon}(s)-V^{0}(s)\right\|^{2} d s+2 C_{\eta} \int_{0}^{t \wedge \tau_{N}}\left|V^{\varepsilon}(s)-V^{0}(s)\right|^{2} \cdot\left|u^{0}(s)\right|_{\mathcal{H}}^{4} d s .
$$

Since $V^{\varepsilon}(s)=\left(u^{\varepsilon}(s)-u^{0}(s)\right) / \sqrt{\varepsilon},(2.3)$ and (2.4) yield

$$
\begin{aligned}
I_{2}(t) & \leq 2 \sqrt{\varepsilon} \int_{0}^{t \wedge \tau_{N}} \eta\left\|V^{\varepsilon}(s)-V^{0}(s)\right\|^{2} d s+2 \sqrt{\varepsilon} C_{\eta} \int_{0}^{t \wedge \tau_{N}}\left|V^{\varepsilon}(s)\right|_{\mathcal{H}}^{4} d s \\
& \leq 2 \sqrt{\varepsilon} \eta \int_{0}^{t \wedge \tau_{N}}\left\|V^{\varepsilon}(s)-V^{0}(s)\right\|^{2} d s+2 \sqrt{\varepsilon} C_{\eta} a_{0} \int_{0}^{t \wedge \tau_{N}}\left|V^{\varepsilon}(s)\right|^{2}\left\|V^{\varepsilon}(s)\right\|^{2} d s .
\end{aligned}
$$

In view of (C4), (C5) and

$$
\begin{aligned}
& \frac{1}{\sqrt{\varepsilon}}\left[\tilde{R}\left(s, u^{\varepsilon}(s)\right)-\tilde{R}\left(s, u^{0}(s)\right)\right]-\tilde{R}^{\prime}\left(s, u^{0}(s)\right) V^{0}(s) \\
& =\frac{1}{\sqrt{\varepsilon}}\left[\tilde{R}\left(s, u^{\varepsilon}(s)\right)-\tilde{R}\left(s, u^{0}(s)\right)\right]+\tilde{R}^{\prime}\left(s, u^{0}(s)\right)\left(\left[V^{\varepsilon}(s)-V^{0}(s)\right]-\frac{1}{\sqrt{\varepsilon}}\left[u^{\varepsilon}(s)-u^{0}(s)\right]\right) \\
& =\tilde{R}^{\prime}\left(s, u^{0}(s)\right)\left[V^{\varepsilon}(s)-V^{0}(s)\right]+\frac{1}{\sqrt{\varepsilon}}\left(\tilde{R}\left(s, u^{\varepsilon}(s)\right)-\tilde{R}\left(s, u^{0}(s)\right)-\tilde{R}^{\prime}\left(s, u^{0}(s)\right)\left[u^{\varepsilon}(s)-u^{0}(s)\right]\right) \\
& =\tilde{R}^{\prime}\left(s, u^{0}(s)\right)\left[V^{\varepsilon}(s)-V^{0}(s)\right] \\
& +\frac{1}{\sqrt{\varepsilon}} \int_{0}^{1}\left[\left(\tilde{R}^{\prime}\left(s, x\left(u^{\varepsilon}(s)-u^{0}(s)\right)+u^{0}(s)\right)-\tilde{R}^{\prime}\left(s, u^{0}(s)\right)\right)\left[u^{\varepsilon}(s)-u^{0}(s)\right]\right] d x, \\
& \text { we obtain } \\
& I_{3}(t) \leq 2 \int_{0}^{t \wedge \tau_{N}}\left(\tilde{R}_{1}^{\prime}+\tilde{R}_{0}^{\prime}\left|u^{0}(s)\right|\right)\left|V^{\varepsilon}(s)-V^{0}(s)\right|^{2} d s+\frac{C}{\sqrt{\varepsilon}} \int_{0}^{t \wedge \tau_{N}}\left|V^{\varepsilon}(s)-V^{0}(s)\right|\left|u^{\varepsilon}(s)-u^{0}(s)\right|^{2} d s
\end{aligned}
$$




$$
\begin{aligned}
& \leq 2 \int_{0}^{t \wedge \tau_{N}}\left(\tilde{R}_{1}^{\prime}+\tilde{R}_{0}^{\prime}\left|u^{0}(s)\right|\right)\left|V^{\varepsilon}(s)-V^{0}(s)\right|^{2} d s+\int_{0}^{t \wedge \tau_{N}}\left|V^{\varepsilon}(s)-V^{0}(s)\right|^{2} d s \\
& +\frac{C^{2}}{\varepsilon} \int_{0}^{t \wedge \tau_{N}}\left|u^{\varepsilon}(s)-u^{0}(s)\right|^{4} d s .
\end{aligned}
$$

For any $\delta>0$, introduce sets $A_{\delta}^{t}=\left\{\omega: \sup _{0 \leq s \leq t}\left|u^{\varepsilon}(s)-u^{0}(s)\right| \leq \delta\right\}$. It is evident that $A_{\delta}^{t}$ is non-increasing w.r.t. $t$. Burkholder-Davis-Gundy inequality and (C2-2) imply

$$
\begin{aligned}
& \mathbb{E}\left(\sup _{0 \leq s \leq t}\left[\mathbf{1}_{A_{\delta}^{s}} I_{4}(s)\right]\right) \\
& \leq 4 \mathbb{E}\left(\int_{0}^{t \wedge \tau_{N}} \mathbf{1}_{A_{\delta}^{s}}\left|\sigma\left(s, u^{\varepsilon}(s)\right)-\sigma\left(s, u^{0}(s)\right)\right|_{L_{Q}}^{2} \cdot\left|V^{\varepsilon}(s)-V^{0}(s)\right|^{2} d s\right)^{\frac{1}{2}} \\
& \leq 4 \mathbb{E}\left(\sup _{0 \leq s \leq t \wedge \tau_{N}}\left[\mathbf{1}_{A_{\delta}^{s}}\left|V^{\varepsilon}(s)-V^{0}(s)\right|\right] \cdot\left(\int_{0}^{t \wedge \tau_{N}} L_{1}\left|u^{\varepsilon}(s)-u^{0}(s)\right|^{2} d s\right)^{\frac{1}{2}}\right) \\
& \leq \frac{1}{2} \mathbb{E}\left(\sup _{0 \leq s \leq t}\left[\mathbf{1}_{A_{\delta}^{s}}\left|V^{\varepsilon}\left(s \wedge \tau_{N}\right)-V^{0}\left(s \wedge \tau_{N}\right)\right|^{2}\right]\right)+8 L_{1} \mathbb{E}\left(\int_{0}^{t \wedge \tau_{N}}\left|u^{\varepsilon}(s)-u^{0}(s)\right|^{2} d s\right) .
\end{aligned}
$$

From (C2-2), we get

$$
I_{5}(t) \leq L_{1} \int_{0}^{t \wedge \tau_{N}}\left|u^{\varepsilon}(s)-u^{0}(s)\right|^{2} d s .
$$

Multiply by $\mathbf{1}_{A_{\delta}^{s}}$, taking the supremum up to time $t$ in (3.6), and then taking the expectation, one obtains

$$
\begin{aligned}
& \frac{1}{2} \mathbb{E}\left(\sup _{0 \leq s \leq t}\left[\mathbf{1}_{A_{\delta}^{s}}\left|V^{\varepsilon}\left(s \wedge \tau_{N}\right)-V^{0}\left(s \wedge \tau_{N}\right)\right|^{2}\right]\right) \\
& +2(1-\eta-\sqrt{\varepsilon} \eta) \mathbb{E}\left(\sup _{0 \leq s \leq t}\left[\mathbf{1}_{A_{\delta}^{s}} \int_{0}^{s \wedge \tau_{N}}\left\|V^{\varepsilon}(l)-V^{0}(l)\right\|^{2} d l\right]\right) \\
& \leq 2 C_{\eta} \mathbb{E}\left(\int_{0}^{t} \sup _{0 \leq l \leq s}\left(\mathbf{1}_{A_{\delta}^{l}}\left|V^{\varepsilon}\left(l \wedge \tau_{N}\right)-V^{0}\left(l \wedge \tau_{N}\right)\right|^{2}\right) \cdot\left|u^{0}(s)\right|_{\mathcal{H}}^{4} d s\right) \\
& +2 \sqrt{\varepsilon} C_{\eta} a_{0} \mathbb{E}\left(\sup _{0 \leq s \leq t}\left(\mathbf{1}_{A_{\delta}^{s}} \int_{0}^{s \wedge \tau_{N}}\left|V^{\varepsilon}(l)\right|^{2}\left\|V^{\varepsilon}(l)\right\|^{2} d l\right)\right) \\
& +\mathbb{E}\left(\int_{0}^{t \wedge \tau_{N}} \mathbf{1}_{A_{\delta}^{s}}|| V^{\varepsilon}(s)-V^{0}(s) \|^{2} d s\right)+2 \mathbb{E}\left(\int_{0}^{t \wedge \tau_{N}} \mathbf{1}_{A_{\delta}^{s}}\left(\tilde{R}_{1}^{\prime}+\tilde{R}_{0}^{\prime}\left|u^{0}(s)\right|\right)\left|V^{\varepsilon}(s)-V^{0}(s)\right|^{2} d s\right) \\
& +\frac{C^{2}}{\varepsilon} \mathbb{E}\left(\sup _{0 \leq s \leq t}\left(\mathbf{1}_{A_{\delta}^{s}} \int_{0}^{s \wedge \tau_{N}}\left|u^{\varepsilon}(l)-u^{0}(l)\right|^{4} d l\right)\right)+8 L_{1} \mathbb{E}\left(\int_{0}^{t \wedge \tau_{N}}\left|u^{\varepsilon}(s)-u^{0}(s)\right|^{2} d s\right) \\
& +L_{1} \mathbb{E}\left(\int_{0}^{t \wedge \tau_{N}}\left|u^{\varepsilon}(s)-u^{0}(s)\right|^{2} d s\right) \\
& \leq \int_{0}^{t} \mathbb{E}\left(\sup _{0 \leq l \leq s}\left[\mathbf{1}_{A_{\delta}^{l}}\left|V^{\varepsilon}\left(l \wedge \tau_{N}\right)-V^{0}\left(l \wedge \tau_{N}\right)\right|^{2}\right) \cdot\left[2 C_{\eta}\left|u^{0}(s)\right|_{\mathcal{H}}^{4}+1+2\left(\tilde{R}_{1}^{\prime}+\tilde{R}_{0}^{\prime}\left|u^{0}(s)\right|\right)\right] d s\right.
\end{aligned}
$$




$$
\begin{aligned}
& +2 \sqrt{\varepsilon} C_{\eta} a_{0} \mathbb{E}\left(\sup _{0 \leq s \leq t}\left(\mathbf{1}_{A_{\delta}^{s}} \int_{0}^{s \wedge \tau_{N}}\left|V^{\varepsilon}(l)\right|^{2}\left\|V^{\varepsilon}(l)\right\|^{2} d l\right)\right) \\
& +C^{2} \delta^{2} \mathbb{E}\left(\int_{0}^{t} \frac{1}{\varepsilon}\left|u^{\varepsilon}(s)-u^{0}(s)\right|^{2} d s\right)+9 L_{1} \mathbb{E}\left(\int_{0}^{t}\left|u^{\varepsilon}(s)-u^{0}(s)\right|^{2} d s\right) \\
& \leq \int_{0}^{t} \mathbb{E}\left(\sup _{0 \leq l \leq s} \mathbf{1}_{A_{\delta}^{l}}\left|V^{\varepsilon}\left(l \wedge \tau_{N}\right)-V^{0}\left(l \wedge \tau_{N}\right)\right|^{2}\right) \cdot\left[2 C_{\eta}\left|u^{0}(s)\right|_{\mathcal{H}}^{4}+1+2\left(\tilde{R}_{1}^{\prime}+\tilde{R}_{0}^{\prime}\left|u^{0}(s)\right|\right)\right] d s \\
& +2 \sqrt{\varepsilon} C_{\eta} a_{0} \cdot C+C^{2} \delta^{2}+C \varepsilon
\end{aligned}
$$

in view of Proposition 3.1 and Lemma 3.2 below. Choosing $\eta+\sqrt{\varepsilon} \eta<1 / 2$, applying the Gronwall's inequality to $\mathbb{E}\left(\sup _{0 \leq s \leq t}\left[\mathbf{1}_{A_{\delta}^{s}}\left|V^{\varepsilon}\left(s \wedge \tau_{N}\right)-V^{0}\left(s \wedge \tau_{N}\right)\right|^{2}\right]\right)$ and taking $N \rightarrow \infty$, it yields

$$
\begin{aligned}
& \mathbb{E}\left(\sup _{0 \leq s \leq t}\left[\mathbf{1}_{A_{\delta}^{s}}\left|V^{\varepsilon}(s)-V^{0}(s)\right|^{2}\right]\right)+\mathbb{E}\left(\sup _{0 \leq s \leq t}\left[\mathbf{1}_{A_{\delta}^{s}} \int_{0}^{s}\left\|V^{\varepsilon}(l)-V^{0}(l)\right\|^{2} d s\right]\right) \\
\leq & C\left(\sqrt{\varepsilon}+\delta^{2}+\varepsilon\right) \exp \left(\int_{0}^{T}\left[2 C_{\eta}\left|u^{0}(s)\right|_{\mathcal{H}}^{4}+1+2\left(\tilde{R}_{1}^{\prime}+\tilde{R}_{0}^{\prime}\left|u^{0}(s)\right|\right)\right] d s\right) .
\end{aligned}
$$

Thus, for any $l>0$,

$$
\begin{aligned}
& \mathbb{P}\left(\sup _{0 \leq t \leq T}\left|V^{\varepsilon}(t)-V^{0}(t)\right|^{2}+\int_{0}^{T}\left\|V^{\varepsilon}(s)-V^{0}(s)\right\|^{2} d s>l\right) \\
\leq & \frac{1}{l} \mathbb{E}\left(\sup _{0 \leq s \leq T}\left[\mathbf{1}_{A_{\delta}^{s}}\left|V^{\varepsilon}(s)-V^{0}(s)\right|^{2}\right]\right) \\
& +\frac{1}{l} \mathbb{E}\left(\sup _{0 \leq s \leq T}\left[\mathbf{1}_{A_{\delta}^{s}} \int_{0}^{s}\left\|V^{\varepsilon}(l)-V^{0}(l)\right\|^{2} d s\right]\right)+\mathbb{P}\left(\left(A_{\delta}^{T}\right)^{c}\right) \\
\leq & \frac{1}{l} C\left(\sqrt{\varepsilon}+\delta^{2}+\varepsilon\right)+\mathbb{P}\left(\left(A_{\delta}^{T}\right)^{c}\right),
\end{aligned}
$$

where $\left(A_{\delta}^{T}\right)^{c}$ denotes the complement of $A_{\delta}^{T}$ and we have used the fact that $A_{\delta}^{t}$ is non-increasing w.r.t. t. By the definition of $A_{\delta}^{t}$ and Proposition $3.1, \mathbb{P}\left(\left(A_{\delta}^{T}\right)^{c}\right) \rightarrow 0$, as $\varepsilon \rightarrow 0$. The arbitrary of $\delta$ implies that $\mathbb{P}\left(\sup _{0 \leq t \leq T}\left|V^{\varepsilon}(t)-V^{0}(t)\right|^{2}+\int_{0}^{T} \| V^{\varepsilon}(s)-\right.$ $\left.V^{0}(s) \|^{2} d s>l\right) \rightarrow 0$, as $\varepsilon \rightarrow 0$ which completes the proof.

For the integrity in the proof of last theorem, we have to show the following estimate. Because the operator $\tilde{R}^{\prime}$ satisfies $(\mathrm{C} 4)$ and (C5), using the similar arguments as the proof of Lemma 3.2 in [19, we have

Lemma 3.2 Under the conditions (C1-1)-(C1-3), (C2-1)-(C2-2) and (C3)-(C5), there exists a constant $\varepsilon_{0}>0$ such that

$$
\sup _{0<\varepsilon \leq \varepsilon_{0}} \mathbb{E}\left(\int_{0}^{T}\left|V^{\varepsilon}(s)\right|^{2} \cdot\left\|V^{\varepsilon}(s)\right\|^{2} d s\right)<\infty .
$$




\section{Moderate deviations}

Let $Z^{\varepsilon}=\left(u^{\varepsilon}-u^{0}\right) /(\sqrt{\varepsilon} \lambda(\varepsilon))$. Then $Z^{\varepsilon}$ satisfies the following SPDE:

$$
\begin{aligned}
& d Z^{\varepsilon}(t)+A Z^{\varepsilon}(t) d t+B\left(Z^{\varepsilon}(t), u^{0}(t)+\sqrt{\varepsilon} \lambda(\varepsilon) Z^{\varepsilon}(t)\right) d t+B\left(u^{0}(t), Z^{\varepsilon}(t)\right) d t \\
& +(\sqrt{\varepsilon} \lambda(\varepsilon))^{-1}\left[\tilde{R}\left(t, u^{0}(t)+\sqrt{\varepsilon} \lambda(\varepsilon) Z^{\varepsilon}(t)\right)-\tilde{R}\left(t, u^{0}(t)\right)\right] d t \\
& =\lambda^{-1}(\varepsilon) \sigma\left(t, u^{0}(t)+\sqrt{\varepsilon} \lambda(\varepsilon) Z^{\varepsilon}(t)\right) d W(t),
\end{aligned}
$$

with initial value $Z^{\varepsilon}(0)=0$. This equation admits a unique solution $Z^{\varepsilon}=\Gamma^{\varepsilon}(W(\cdot))$, where $\Gamma^{\varepsilon}$ stands for the solution functional from $C([0, T] ; H)$ into $C([0, T] ; H) \cap$ $L^{2}([0, T] ; V)$.

In this part, we will prove that $Z^{\varepsilon}$ satisfies an LDP on $C([0, T] ; H) \cap L^{2}([0, T] ; V)$ with $\lambda(\varepsilon)$ satisfying (1.4). This special type of LDP is usually called the MDP of $u^{\varepsilon}$ (cf. 8 ).

Firstly we recall the general criteria for a large deviation principle (LDP) given in 2. Let $\mathcal{E}$ be a Polish space with the Borel $\sigma$-field $\mathcal{B}(\mathcal{E})$.

Definition 4.1 (Rate function) A function $I: \mathcal{E} \rightarrow[0, \infty]$ is called a rate function on $\mathcal{E}$, if for each $M<\infty$, the level set $\{x \in \mathcal{E}: I(x) \leq M\}$ is a compact subset of $\mathcal{E}$.

Definition 4.2 (LDP) Let I be a rate function on $\mathcal{E}$. A family $\left\{X^{\varepsilon}\right\}$ of $\mathcal{E}$-valued random elements is said to satisfy the $L D P$ on $\mathcal{E}$ with rate function $I$, if the following two conditions hold.

(a) (Large deviation upper bound) For each closed subset $F$ of $\mathcal{E}$,

$$
\limsup _{\varepsilon \rightarrow 0} \varepsilon \log \mathbb{P}\left(X^{\varepsilon} \in F\right) \leq-\inf _{x \in F} I(x) .
$$

(b) (Large deviation lower bound) For each open subset $G$ of $\mathcal{E}$,

$$
\liminf _{\varepsilon \rightarrow 0} \varepsilon \log \mathbb{P}\left(X^{\varepsilon} \in G\right) \geq-\inf _{x \in G} I(x) .
$$

Next, we introduce the Skeleton Equations. The Cameron-Martin space associated with the Wiener process $\{W(t), t \in[0, T]\}$ is given by

$$
\mathcal{H}_{0}:=\left\{h:[0, T] \rightarrow H_{0} ; h \text { is absolutely continuous and } \int_{0}^{T}|\dot{h}(s)|_{0}^{2} d s<+\infty\right\} .
$$

The space $\mathcal{H}_{0}$ is a Hilbert space with inner product $\left\langle h_{1}, h_{2}\right\rangle_{\mathcal{H}_{0}}:=$ $\int_{0}^{T}\left\langle\dot{h}_{1}(s), \dot{h}_{2}(s)\right\rangle_{0} d s$. Let $\mathcal{A}$ denote the class of $H_{0}$-valued $\left\{\mathcal{F}_{t}\right\}$-predictable processes $\phi$ belonging to $\mathcal{H}_{0}$ a.s.. Let $S_{N}=\left\{h \in \mathcal{H}_{0} ; \int_{0}^{T}|\dot{h}(s)|_{0}^{2} d s \leq N\right\}$. The set $S_{N}$ endowed with the weak topology is a Polish space. Define $\mathcal{A}_{N}=\left\{\phi \in \mathcal{A} ; \phi(\omega) \in S_{N}\right.$, $\mathbb{P}$-a.s. $\}$. 
For any $h \in \mathcal{H}_{0}$, consider the deterministic integral equation

$$
\begin{aligned}
& d X^{h}(t)+\left(A X^{h}(t)+B\left(X^{h}(t), u^{0}(t)\right)+B\left(u^{0}(t), X^{h}(t)\right)\right) d t \\
& +\tilde{R}^{\prime}\left(t, u^{0}(t)\right) \cdot X^{h}(t) d t=\sigma\left(t, u^{0}(t)\right) \dot{h}(t) d t
\end{aligned}
$$

with initial value $X^{h}(0)=0$ and for any $\phi^{\varepsilon} \in \mathcal{A}$, consider

$$
\begin{aligned}
& d X^{\varepsilon}(t)+A X^{\varepsilon}(t) d t+B\left(X^{\varepsilon}(t), u^{0}(t)+\sqrt{\varepsilon} \lambda(\varepsilon) X^{\varepsilon}(t)\right) d t+B\left(u^{0}(t), X^{\varepsilon}(t)\right) d t \\
& +(\sqrt{\varepsilon} \lambda(\varepsilon))^{-1}\left[\tilde{R}\left(t, u^{0}(t)+\sqrt{\varepsilon} \lambda(\varepsilon) X^{\varepsilon}(t)\right)-\tilde{R}\left(t, u^{0}(t)\right)\right] d t \\
& =\lambda^{-1}(\varepsilon) \sigma\left(t, u^{0}(t)+\sqrt{\varepsilon} \lambda(\varepsilon) X^{\varepsilon}(t)\right) d W(t)+\sigma\left(t, u^{0}(t)+\sqrt{\varepsilon} \lambda(\varepsilon) X^{\varepsilon}(t)\right) \dot{\phi}^{\varepsilon}(t) d t,
\end{aligned}
$$

with initial value $X^{\varepsilon}(0)=0$.

Now we are ready to state the second main result. We assume that (C6) there exist $\kappa, C>0$, for any $t_{1}, t_{2} \in[0, T], u \in V$,

$$
\left|\sigma\left(t_{1}, u\right)-\sigma\left(t_{2}, u\right)\right|_{L_{Q}} \leq C(1+\|u\|)\left|t_{1}-t_{2}\right|^{\kappa} .
$$

Theorem 4.3 (Moderate Deviation Principle) Under the conditions (C1-1)(C1-3), (C2-1)-(C2-2) and (C3)-(C6), $\left(u^{\varepsilon}-u^{0}\right) /(\sqrt{\varepsilon} \lambda(\varepsilon))$ obeys an LDP on $C([0, T] ; H) \cap L^{2}([0, T] ; V)$ with speed $\lambda^{2}(\varepsilon)$ and with rate function I given by

$$
I(g):=\inf _{\left\{h \in \mathcal{H}_{0} ; g=X^{h}\right\}}\left\{\frac{1}{2} \int_{0}^{T}|\dot{h}(s)|_{0}^{2} d s\right\}, \quad \forall g \in C([0, T] ; H) \cap L^{2}([0, T] ; V),
$$

with the convention $\inf \{\emptyset\}=\infty$.

We will adopt the following weak convergence method to prove the MDP.

Theorem 4.4 (Budhiraja and Dupuis[2) For $\varepsilon>0$, let $\Gamma^{\varepsilon}$ be a measurable mapping from $C([0, T] ; H)$ into $\mathcal{E}$. Let $Y^{\varepsilon}:=\Gamma^{\varepsilon}(W(\cdot))$. Suppose that $\left\{\Gamma^{\varepsilon}\right\}_{\varepsilon>0}$ satisfies the following assumptions: there exists a measurable map $\Gamma^{0}: C([0, T] ; H) \rightarrow \mathcal{E}$ such that

(a) for every $N<+\infty$ and any family $\left\{h^{\varepsilon} ; \varepsilon>0\right\} \subset \mathcal{A}_{N}$ satisfying that $h^{\varepsilon}$ converge in distribution as $S_{N}$-valued random elements to $h$ as $\varepsilon \rightarrow$ $0, \Gamma^{\varepsilon}\left(W(\cdot)+\frac{1}{\sqrt{\varepsilon}} \int_{0}^{\cdot} \dot{h}^{\varepsilon}(s) d s\right)$ converges in distribution to $\Gamma^{0}\left(\int_{0}^{\cdot} \dot{h}(s) d s\right)$ as $\varepsilon \rightarrow 0$

(b) for every $N<+\infty$, the set $\left\{\Gamma^{0}\left(\int_{0}^{\cdot} \dot{h}(s) d s\right) ; h \in S_{N}\right\}$ is a compact subset of $\mathcal{E}$.

Then the family $\left\{Y^{\varepsilon}\right\}_{\varepsilon>0}$ satisfies a LDP in $\mathcal{E}$ with the rate function I given by

$$
I(g):=\inf _{\left\{h \in \mathcal{H}_{0} ; g=\Gamma^{0}\left(\int_{0}^{\cdot} \dot{h}(s) d s\right)\right\}}\left\{\frac{1}{2} \int_{0}^{T}|\dot{h}(s)|_{0}^{2} d s\right\}, g \in \mathcal{E},
$$


with the convention $\inf \{\emptyset\}=\infty$.

For $h \in \mathcal{H}_{0}$, set $\Gamma^{0}\left(\int_{0} \dot{h}(s) d s\right):=X^{h}$ and define $\Gamma^{\varepsilon}$ satisfies $\Gamma^{\varepsilon}\left(W(\cdot)+\frac{\lambda(\varepsilon)}{\sqrt{\varepsilon}} \int_{0}^{\cdot} \dot{\phi}^{\varepsilon}(s) d s\right)=X^{\varepsilon}$. To prove Theorem 4.3, we only need to verify the following two propositions according to Theorem 4.4

Proposition 4.5 Under the same conditions as Theorem 4.3, for every fixed $N \in$ $\mathbb{N}$, let $\phi^{\varepsilon}, \phi \in \mathcal{A}_{N}$ be such that $\phi^{\varepsilon}$ convergence in distribution to $\phi$ as $\varepsilon \rightarrow 0$. Then

$$
\Gamma^{\varepsilon}\left(W(\cdot)+\frac{\lambda(\varepsilon)}{\sqrt{\varepsilon}} \int_{0}^{\cdot} \dot{\phi}^{\varepsilon}(s) d s\right) \text { convergence in distribution to } \Gamma^{0}\left(\int_{0}^{\cdot} \dot{\phi}(s) d s\right)
$$

in $C([0, T] ; H) \cap L^{2}([0, T] ; V)$ as $\varepsilon \rightarrow 0$.

Proposition 4.6 Under the same conditions as Theorem 4.3, for every positive number $N<\infty$, the family $K_{N}:=\left\{\Gamma^{0}\left(\int_{0}^{\cdot} \dot{h}(s) d s\right) ; h \in S_{N}\right\}$ is compact in $C([0, T] ; H) \cap L^{2}([0, T] ; V)$.

We start to prove Proposition 4.5 and we need the following two lemmas. In this section, set $B(u, v, w)=(B(u, v), w)$.

Lemma 4.1 Under the same conditions as Theorem 4.3, for any $h \in \mathcal{H}_{0}$ and $\phi^{\varepsilon} \in$ $\mathcal{A}$, (4.3) and (4.4) admit the unique solutions, respectively, $X^{h}, X^{\varepsilon}$ in $C([0, T] ; H) \cap$ $L^{2}([0, T] ; V)$. Moreover, for any $N>0$ and $p \geq 2$, there exist constants $c_{T, N}$ and $\varepsilon_{0}>0$ such that for any $h \in S_{N}, \phi^{\varepsilon} \in \mathcal{A}_{N}$,

$$
\sup _{\varepsilon \in\left(0, \varepsilon_{0}\right]}\left[\mathbb{E}\left(\sup _{0 \leq t \leq T}\left|X^{\varepsilon}(t)\right|^{2 p}\right)+\mathbb{E}\left(\int_{0}^{T}\left|X^{\varepsilon}(s)\right|^{2 p-2}\left\|X^{\varepsilon}(s)\right\|^{2} d s\right)\right] \leq c_{T, N}
$$

and specially,

$$
\sup _{0 \leq t \leq T}\left|X^{h}(t)\right|^{2 p}+\int_{0}^{T}\left|X^{h}(s)\right|^{2 p-2}\left\|X^{h}(s)\right\|^{2} d s \leq c_{T, N} .
$$

Proof. Similarly as Theorem 2.4 in 7 , the existence and uniqueness of the solution can be proved. Here, we will prove (4.8) and (4.9).

Define $\tau_{M}:=\inf \left\{t:\left|X^{\varepsilon}(t)\right|^{2}+\int_{0}^{t}\left\|X^{\varepsilon}(s)\right\|^{2} d s>M\right\}$. By Itô's formula and (4.4), we have

$$
\begin{aligned}
& \left|X^{\varepsilon}\left(t \wedge \tau_{M}\right)\right|^{2}+2 \int_{0}^{t \wedge \tau_{M}}\left\|X^{\varepsilon}(s)\right\|^{2} d s+2 \int_{0}^{t \wedge \tau_{M}}\left(B\left(X^{\varepsilon}(s), u^{0}(s)\right), X^{\varepsilon}(s)\right) d s \\
& =-2(\sqrt{\varepsilon} \lambda(\varepsilon))^{-1} \int_{0}^{t \wedge \tau_{M}}\left(\tilde{R}\left(s, u^{0}(s)+\sqrt{\varepsilon} \lambda(\varepsilon) X^{\varepsilon}(t)\right)-\tilde{R}\left(s, u^{0}(s)\right), X^{\varepsilon}(s)\right) d s \\
& +2 \lambda^{-1}(\varepsilon) \int_{0}^{t \wedge \tau_{M}}\left(\sigma\left(s, u^{0}(s)+\sqrt{\varepsilon} \lambda(\varepsilon) X^{\varepsilon}(s)\right) d W(s), X^{\varepsilon}(s)\right)
\end{aligned}
$$




$$
\begin{aligned}
& +2 \int_{0}^{t \wedge \tau_{M}}\left(\sigma\left(s, u^{0}(s)+\sqrt{\varepsilon} \lambda(\varepsilon) X^{\varepsilon}(s)\right) \dot{\phi}^{\varepsilon}(s), X^{\varepsilon}(s)\right) d s \\
& +\lambda^{-2}(\varepsilon) \int_{0}^{t \wedge \tau_{M}}\left|\sigma\left(s, u^{0}(s)+\sqrt{\varepsilon} \lambda(\varepsilon) X^{\varepsilon}(s)\right)\right|_{L_{Q}}^{2} d s .
\end{aligned}
$$

Applying Itô's formula to $f(x)=x^{p}$ with $x=\left|X^{\varepsilon}\left(t \wedge \tau_{M}\right)\right|^{2}$, we have $d f(x)=$ $p x^{p-1} d x+\frac{1}{2} p(p-1) x^{p-2} d<x>$. Then

$$
\begin{aligned}
& \left|X^{\varepsilon}\left(t \wedge \tau_{M}\right)\right|^{2 p} \\
= & \int_{0}^{t \wedge \tau_{M}} p\left|X^{\varepsilon}(s)\right|^{2(p-1)} d\left|X^{\varepsilon}(s)\right|^{2}+\frac{1}{2} p(p-1) \int_{0}^{t \wedge \tau_{M}}\left|X^{\varepsilon}(s)\right|^{2(p-2)} d<\left|X^{\varepsilon}\right|^{2}>(s) \\
= & -2 p \int_{0}^{t \wedge \tau_{M}}\left|X^{\varepsilon}(s)\right|^{2(p-1)}|| X^{\varepsilon}(s) \|^{2} d s-2 p \int_{0}^{t \wedge \tau_{M}}\left|X^{\varepsilon}(s)\right|^{2(p-1)}\left(B\left(X^{\varepsilon}(s), u^{0}(s)\right), X^{\varepsilon}(s)\right) d s \\
& -2 p(\sqrt{\varepsilon} \lambda(\varepsilon))^{-1} \int_{0}^{t \wedge \tau_{M}}\left|X^{\varepsilon}(s)\right|^{2(p-1)}\left(\tilde{R}\left(s, u^{0}(s)+\sqrt{\varepsilon} \lambda(\varepsilon) X^{\varepsilon}(t)\right)-\tilde{R}\left(s, u^{0}(s)\right), X^{\varepsilon}(s)\right) d s \\
& +2 p \lambda^{-1}(\varepsilon) \int_{0}^{t \wedge \tau_{M}}\left|X^{\varepsilon}(s)\right|^{2(p-1)}\left(\sigma\left(s, u^{0}(s)+\sqrt{\varepsilon} \lambda(\varepsilon) X^{\varepsilon}(s)\right) d W(s), X^{\varepsilon}(s)\right) \\
& +2 p \int_{0}^{t \wedge \tau_{M}}\left|X^{\varepsilon}(s)\right|^{2(p-1)}\left(\sigma\left(s, u^{0}(s)+\sqrt{\varepsilon} \lambda(\varepsilon) X^{\varepsilon}(s)\right) \dot{\phi}^{\varepsilon}(s), X^{\varepsilon}(s)\right) d s \\
& +p \lambda^{-2}(\varepsilon) \int_{0}^{t \wedge \tau_{M}}\left|X^{\varepsilon}(s)\right|^{2(p-1)}\left|\sigma\left(s, u^{0}(s)+\sqrt{\varepsilon} \lambda(\varepsilon) X^{\varepsilon}(s)\right)\right|_{L_{Q}}^{2} d s \\
& +\frac{1}{2} p(p-1) \cdot 4 \lambda^{-2}(\varepsilon) \int_{0}^{t \wedge \tau_{M}}\left|X^{\varepsilon}(s)\right|^{2(p-2)}\left|\left(\sigma\left(s, u^{0}(s)+\sqrt{\varepsilon} \lambda(\varepsilon) X^{\varepsilon}(s)\right), X^{\varepsilon}(s)\right)\right|_{L_{Q}\left(H_{0}, R\right)}^{2} d s \\
:= & \sum_{k=1}^{7} I_{k} .
\end{aligned}
$$

By (2.7), one has

$$
\left|I_{2}(t)\right| \leq 2 p \int_{0}^{t \wedge \tau_{M}}\left|X^{\varepsilon}(s)\right|^{2(p-1)}\left(\eta\left\|X^{\varepsilon}(s)\right\|^{2}+C_{\eta}\left|X^{\varepsilon}(s)\right|^{2} \cdot\left\|u^{0}(s)\right\|_{\mathcal{H}}^{4}\right) d s .
$$

From (C3), we have

$$
\left|I_{3}(t)\right| \leq 2 p R_{1} \int_{0}^{t \wedge \tau_{M}}\left|X^{\varepsilon}(s)\right|^{2 p} d s .
$$

By the Burkholder-Davis-Gundy inequality and (C2-1), we have

$$
\begin{aligned}
& \mathbb{E}\left(\sup _{0 \leq s \leq t}\left|I_{4}(s)\right|\right) \\
= & 2 p \lambda^{-1}(\varepsilon) \mathbb{E}\left(\left.\sup _{0 \leq s \leq t}\left|\int_{0}^{s \wedge \tau_{M}}\right| X^{\varepsilon}(l)\right|^{2(p-1)}\left(\sigma\left(l, u^{0}(l)+\sqrt{\varepsilon} \lambda(\varepsilon) X^{\varepsilon}(l)\right) d W(l), X^{\varepsilon}(l)\right) \mid\right) \\
\leq & 2 p \lambda^{-1}(\varepsilon) \mathbb{E}\left(\int_{0}^{t \wedge \tau_{M}}\left|X^{\varepsilon}(s)\right|^{2(p-1) \times 2+2}\left|\sigma\left(s, u^{0}(s)+\sqrt{\varepsilon} \lambda(\varepsilon) X^{\varepsilon}(s)\right)\right|_{L_{Q}}^{2} d s\right)^{1 / 2}
\end{aligned}
$$




$$
\begin{aligned}
\leq & 2 p \lambda^{-1}(\varepsilon) \mathbb{E}\left(\sup _{0 \leq s \leq t}\left|X^{\varepsilon}\left(s \wedge \tau_{M}\right)\right|^{p}\right. \\
& \left.\cdot\left[\int_{0}^{t \wedge \tau_{M}}\left|X^{\varepsilon}(s)\right|^{2 p-2}\left(K_{0}+2 K_{1}\left|u^{0}(s)\right|^{2}+2 K_{1} \varepsilon \lambda^{2}(\varepsilon)\left|X^{\varepsilon}(s)\right|^{2}\right) d s\right]^{\frac{1}{2}}\right) \\
\leq & 1 / 4 \mathbb{E}\left(\sup _{0 \leq s \leq t}\left|X^{\varepsilon}\left(s \wedge \tau_{M}\right)\right|^{2 p}\right)+c_{p} \lambda^{-2}(\varepsilon) \mathbb{E}\left(\int_{0}^{t \wedge \tau_{M}}\left|X^{\varepsilon}(s)\right|^{2 p-2}\left(K_{0}+2 K_{1}\left|u^{0}(s)\right|^{2}\right) d s\right) \\
& +c_{p, K_{1}} \varepsilon \mathbb{E}\left(\int_{0}^{t \wedge \tau_{M}}\left|X^{\varepsilon}(s)\right|^{2 p} d s\right) \\
\leq & 1 / 4 \mathbb{E}\left(\sup _{0 \leq s \leq t}\left|X^{\varepsilon}\left(s \wedge \tau_{M}\right)\right|^{2 p}\right)+c_{p} \lambda^{-2}(\varepsilon)\left(K_{0}+2 K_{1} \sup _{0 \leq s \leq T}\left|u^{0}(s)\right|^{2}\right) \\
& \cdot \mathbb{E}\left(\int_{0}^{t \wedge \tau_{M}} 1+\left|X^{\varepsilon}(s)\right|^{2 p} d s\right)+c_{p, K_{1}} \varepsilon \mathbb{E}\left(\int_{0}^{t \wedge \tau_{M}}\left|X^{\varepsilon}(s)\right|^{2 p} d s\right) \\
\leq & \left(1 / 4+c_{p, K_{0}, K_{1}, T}\left(\lambda^{-2}(\varepsilon)+\varepsilon\right)\right) \mathbb{E}\left(\sup _{0 \leq s \leq t}\left|X^{\varepsilon}\left(s \wedge \tau_{M}\right)\right|^{2 p}\right)+c_{p, K_{0}, K_{1}, T} \lambda^{-2}(\varepsilon) .
\end{aligned}
$$

Because of

$$
\begin{aligned}
\left|\sigma\left(s, u^{0}(s)+\sqrt{\varepsilon} \lambda(\varepsilon) X^{\varepsilon}(s)\right)\right|_{L_{Q}} & \leq\left(K_{0}+K_{1}\left|u^{0}(s)+\sqrt{\varepsilon} \lambda(\varepsilon) X^{\varepsilon}(s)\right|^{2}\right)^{\frac{1}{2}} \\
& \leq \sqrt{K_{0}}+\sqrt{K_{1}}\left|u^{0}(s)+\sqrt{\varepsilon} \lambda(\varepsilon) X^{\varepsilon}(s)\right| \\
& \leq \sqrt{K_{0}}+\sqrt{K_{1}}\left|u^{0}(s)\right|+\sqrt{K_{1}} \sqrt{\varepsilon} \lambda(\varepsilon)\left|X^{\varepsilon}(s)\right|,
\end{aligned}
$$

we get

$$
\begin{aligned}
&\left|I_{5}(t)\right| \\
& \leq 2 p \int_{0}^{t \wedge \tau_{M}}\left|X^{\varepsilon}(s)\right|^{2(p-1)}\left|X^{\varepsilon}(s)\right| \cdot\left|\sigma\left(s, u^{0}(s)+\sqrt{\varepsilon} \lambda(\varepsilon) X^{\varepsilon}(s)\right)\right| L_{Q}\left|\dot{\phi}^{\varepsilon}(s)\right| d s \\
& \leq 2 p \int_{0}^{t \wedge \tau_{M}}\left|X^{\varepsilon}(s)\right|^{2 p-1}\left(\sqrt{K_{0}}+\sqrt{K_{1}}\left|u^{0}(s)\right|\right)\left|\dot{\phi}^{\varepsilon}(s)\right| d s \\
&+2 p \sqrt{K_{1}} \sqrt{\varepsilon} \lambda(\varepsilon) \int_{0}^{t \wedge \tau_{M}}\left|X^{\varepsilon}(s)\right|^{2 p}\left|\dot{\phi}^{\varepsilon}(s)\right| d s \\
& \leq 2 p \int_{0}^{t \wedge \tau_{M}}\left(1+\left|X^{\varepsilon}(s)\right|^{2 p}\right)\left(\sqrt{K_{0}}+\sqrt{K_{1}}\left|u^{0}(s)\right|\right)\left|\dot{\phi}^{\varepsilon}(s)\right| d s \\
&+2 p \sqrt{K_{1}} \sqrt{\varepsilon} \lambda(\varepsilon) \int_{0}^{t \wedge \tau_{M}}\left|X^{\varepsilon}(s)\right|^{2 p}\left|\dot{\phi}^{\varepsilon}(s)\right| d s \\
& \leq c_{p, K_{0}, K_{1}, T, N}+2 p \int_{0}^{t \wedge \tau_{M}}\left|X^{\varepsilon}(s)\right|^{2 p}\left(\sqrt{K_{0}}+\sqrt{K_{1}}\left|u^{0}(s)\right|\right)\left|\dot{\phi}^{\varepsilon}(s)\right| d s \\
&+c_{p, K_{1}, N, T} \sqrt{\varepsilon} \lambda(\varepsilon)\left(\sup _{0 \leq s \leq t}\left|X^{\varepsilon}\left(s \wedge \tau_{M}\right)\right|^{2 p}\right) \\
&\left|I_{6}(t)\right|+\left|I_{7}(t)\right|
\end{aligned}
$$




$$
\begin{aligned}
& \leq\left(p \lambda^{-2}(\varepsilon)+2 p(p-1) \lambda^{-2}(\varepsilon)\right) \int_{0}^{t \wedge \tau_{M}}\left|X^{\varepsilon}(s)\right|^{2(p-1)}\left|\sigma\left(s, u^{0}(s)+\sqrt{\varepsilon} \lambda(\varepsilon) X^{\varepsilon}(s)\right)\right|_{L_{Q}}^{2} d s \\
& \leq\left(p \lambda^{-2}(\varepsilon)+2 p(p-1) \lambda^{-2}(\varepsilon)\right) \int_{0}^{t \wedge \tau_{M}}\left|X^{\varepsilon}(s)\right|^{2(p-1)}\left(K_{0}+2 K_{1}\left|u^{0}(s)\right|^{2}+2 K_{1} \varepsilon \lambda^{2}(\varepsilon)\left|X^{\varepsilon}(s)\right|^{2}\right) d s \\
& \leq c_{p, K_{0}, K_{1}, T}\left(\lambda^{-2}(\varepsilon)+\varepsilon\right)\left(\sup _{0 \leq s \leq t}\left|X^{\varepsilon}\left(s \wedge \tau_{M}\right)\right|^{2 p}\right)+c_{p, K_{0}, K_{1}, T} \lambda^{-2}(\varepsilon) .
\end{aligned}
$$

Taking the supremum up to time $t$ in (4.10), and then taking the expectation, Gronwall's inequality implies

$$
\begin{aligned}
& \left(3 / 4-c\left(\lambda^{-2}(\varepsilon)+\varepsilon+\sqrt{\varepsilon} \lambda(\varepsilon)\right) \mathbb{E}\left(\sup _{0 \leq s \leq t}\left|X^{\varepsilon}\left(s \wedge \tau_{M}\right)\right|^{2 p}\right)\right. \\
& +(2 p-2 p \eta) \mathbb{E}\left(\int_{0}^{t \wedge \tau_{M}}\left|X^{\varepsilon}(s)\right|^{2(p-1)}\left\|X^{\varepsilon}(s)\right\|^{2} d s\right) \\
\leq & C \exp \left[\int_{0}^{T}\left(2 p C_{\eta}\left\|u^{0}(s)\right\|_{\mathcal{H}}^{4}+2 p R_{1}+2 p\left(\sqrt{K}_{0}+\sqrt{K}_{1}\left|u^{0}(s)\right|\right)\left|\dot{\phi}^{\varepsilon}(s)\right|\right) d s\right] \\
\leq & C_{N},
\end{aligned}
$$

for any $\phi^{\varepsilon} \in \mathcal{A}_{N}$, here we choose $\varepsilon$ and $\eta$ small enough. Letting $M \rightarrow \infty$, we get (4.8).

The proof of (4.9) is very similar to that of the deterministic case (4.8), we omit it here. The proof of this lemma is complete.

For every integer $n$, let $\psi_{n}:[0, T] \rightarrow[0, T]$ denote a measurable map such that for every $s \in[0, T], s \leq \psi_{n}(s) \leq\left(s+c 2^{-n}\right) \wedge T$ for some positive constant $c$. Let $G_{M}^{\varepsilon}(t)=\left\{\omega:\left(\sup _{0 \leq s \leq t}\left|X^{\varepsilon}(s)\right|^{2}\right) \vee\left(\int_{0}^{t}\left\|X^{\varepsilon}(s)\right\|^{2} d s\right) \leq M\right\}$. We show the following lemma.

Lemma 4.2 There exist $\varepsilon_{0}, N_{0}>0$ such that for any $\varepsilon \in\left(0, \varepsilon_{0}\right]$ and $\phi^{\varepsilon} \in \mathcal{A}_{N}$,

$$
\mathbb{E}\left(\mathbf{1}_{G_{M}^{\varepsilon}(T)} \int_{0}^{T}\left|X^{\varepsilon}\left(\psi_{n}(s)\right)-X^{\varepsilon}(s)\right|^{2} d s\right) \leq C M^{3} N 2^{-n / 2},
$$

for any $M>N_{0}$, where $C$ is independent on $\varepsilon, M$ and $N$.

Proof. Letting $\sup _{0 \leq s \leq T}\left|u^{0}(s)\right|^{2}+\int_{0}^{T}\left\|u^{0}(s)\right\|^{2} d s \leq N_{0}$. Itô's formula yields

$$
\begin{aligned}
& \mathbb{E}\left(\mathbf{1}_{G_{M}^{\varepsilon}(T)} \int_{0}^{T}\left|X^{\varepsilon}\left(\psi_{n}(s)\right)-X^{\varepsilon}(s)\right|^{2} d s\right) \\
= & \mathbb{E}\left(\mathbf{1}_{G_{M}^{\varepsilon}(T)} \int_{0}^{T} 2 \int_{s}^{\psi_{n}(s)}\left(X^{\varepsilon}(r)-X^{\varepsilon}(s), d X^{\varepsilon}(r)\right) d r d s\right) \\
& +\mathbb{E}\left(\mathbf{1}_{G_{M}^{\varepsilon}(T)} \int_{0}^{T} \int_{s}^{\psi_{n}(s)} \lambda^{-2}(\varepsilon)\left|\sigma\left(r, \sqrt{\varepsilon} \lambda(\varepsilon) X^{\varepsilon}(r)+u^{0}(r)\right)\right|_{L_{Q}}^{2} d r d s\right)
\end{aligned}
$$




$$
\begin{aligned}
= & 2 \mathbb{E}\left(\mathbf{1}_{G_{M}^{\varepsilon}(T)} \int_{0}^{T} \int_{s}^{\psi_{n}(s)}\left(X^{\varepsilon}(r)-X^{\varepsilon}(s),-A X^{\varepsilon}(r)\right) d r d s\right) \\
& -2 \mathbb{E}\left(\mathbf{1}_{G_{M}^{\varepsilon}(T)} \int_{0}^{T} \int_{s}^{\psi_{n}(s)}\left(X^{\varepsilon}(r)-X^{\varepsilon}(s), B\left(X^{\varepsilon}(r), \sqrt{\varepsilon} \lambda(\varepsilon) X^{\varepsilon}(r)+u^{0}(r)\right)\right) d r d s\right) \\
& -2 \mathbb{E}\left(\mathbf{1}_{G_{M}^{\varepsilon}(T)} \int_{0}^{T} \int_{s}^{\psi_{n}(s)}\left(X^{\varepsilon}(r)-X^{\varepsilon}(s), B\left(u^{0}(r), X^{\varepsilon}(r)\right)\right) d r d s\right) \\
& -2 \mathbb{E} \mathbf{1}_{G_{M}^{\varepsilon}(T)} \int_{0}^{T} \int_{s}^{\psi_{n}(s)} \frac{1}{\sqrt{\varepsilon} \lambda(\varepsilon)} \\
& \cdot\left(X^{\varepsilon}(r)-X^{\varepsilon}(s), \tilde{R}\left(r, \sqrt{\varepsilon} \lambda(\varepsilon) X^{\varepsilon}(r)+u^{0}(r)\right)-\tilde{R}\left(r, u^{0}(r)\right)\right) d r d s \\
& +2 \mathbb{E}\left(\mathbf{1}_{G_{M}^{\varepsilon}(T)} \int_{0}^{T} \int_{s}^{\psi_{n}(s)} \lambda^{-1}(\varepsilon)\left(X^{\varepsilon}(r)-X^{\varepsilon}(s), \sigma\left(r, \sqrt{\varepsilon} \lambda(\varepsilon) X^{\varepsilon}(r)+u^{0}(r)\right) d W(r)\right) d s\right) \\
& +2 \mathbb{E}\left(\mathbf{1}_{G_{M}^{\varepsilon}(T)} \int_{0}^{T} \int_{s}^{\psi_{n}(s)}\left(X^{\varepsilon}(r)-X^{\varepsilon}(s), \sigma\left(r, \sqrt{\varepsilon} \lambda(\varepsilon) X^{\varepsilon}(r)+u^{0}(r)\right) \dot{\phi}^{\varepsilon}(r)\right) d r d s\right) \\
& +\mathbb{E}\left(\mathbf{1}_{G_{M}^{\varepsilon}(T)} \int_{0}^{T} \int_{s}^{\psi_{n}(s)} \lambda^{-2}(\varepsilon)\left|\sigma\left(r, \sqrt{\varepsilon} \lambda(\varepsilon) X^{\varepsilon}(r)+u^{0}(r)\right)\right|_{L_{Q}}^{2} d r d s\right) \\
= & \sum_{k=1}^{7} I_{k} .
\end{aligned}
$$

Now we estimate $I_{k}$, respectively.

$$
\begin{aligned}
I_{1} & =2 \mathbb{E}\left(\mathbf{1}_{G_{M}^{\varepsilon}(T)} \int_{0}^{T} \int_{s}^{\psi_{n}(s)}-\left\|X^{\varepsilon}(r)\right\|^{2}+\left\|X^{\varepsilon}(s)\right\|\left\|X^{\varepsilon}(r)\right\| d r d s\right) \\
& \leq 1 / 2 \mathbb{E}\left(\mathbf{1}_{G_{M}^{\varepsilon}(T)} \int_{0}^{T}\left\|X^{\varepsilon}(s)\right\|^{2} d s \int_{s}^{\psi_{n}(s)} d r\right) \\
& \leq C \cdot M \cdot 2^{-(n+1)} .
\end{aligned}
$$

For $I_{2}$,

$$
\begin{aligned}
I_{2} \leq & 2 \mathbb{E}\left(\mathbf{1}_{G_{M}^{\varepsilon}(T)} \int_{0}^{T} \int_{s}^{\psi_{n}(s)}\left|B\left(X^{\varepsilon}(r), u^{0}(r), X^{\varepsilon}(r)\right)\right| d r d s\right) \\
& +2 \mathbb{E}\left(\mathbf{1}_{G_{M}^{\varepsilon}(T)} \int_{0}^{T} \int_{s}^{\psi_{n}(s)}\left|B\left(X^{\varepsilon}(r), \sqrt{\varepsilon} \lambda(\varepsilon) X^{\varepsilon}(r), X^{\varepsilon}(s)\right)\right| d r d s\right) \\
& +2 \mathbb{E}\left(\mathbf{1}_{G_{M}^{\varepsilon}(T)} \int_{0}^{T} \int_{s}^{\psi_{n}(s)}\left|B\left(X^{\varepsilon}(r), u^{0}(r), X^{\varepsilon}(s)\right)\right| d r d s\right) \\
=: & J_{1}+J_{3}+J_{3}, \text { where }
\end{aligned}
$$




$$
\begin{aligned}
& J_{1} \leq 2 \mathbb{E}\left(\mathbf{1}_{G_{M}^{\varepsilon}(T)} \int_{0}^{T} \int_{s}^{\psi_{n}(s)} \eta\left\|X^{\varepsilon}(r)\right\|^{2}+C_{\eta}\left|X^{\varepsilon}(r)\right|^{2}\left\|u^{0}(r)\right\|_{\mathcal{H}}^{4} d r d s\right) \\
& \leq 2 \eta \mathbb{E}\left(\mathbf{1}_{G_{M}^{\varepsilon}(T)} \int_{0}^{T}\left\|X^{\varepsilon}(r)\right\|^{2} d r \int_{\left(r-c 2^{-n}\right) \vee 0}^{r} d s\right) \\
&+2 C_{\eta} a_{0}^{2} \mathbb{E}\left(\mathbf{1}_{G_{M}^{\varepsilon}(T)} \int_{0}^{T} \int_{s}^{\psi_{n}(s)}\left|X^{\varepsilon}(r)\right|^{2} \cdot\left|u^{0}(r)\right|^{2}\left\|u^{0}(r)\right\|^{2} d r d s\right) \\
& \leq 2 \eta C 2^{-n} M+2 C_{\eta} \cdot a_{0}^{2} \cdot M^{2} \mathbb{E}\left(\mathbf{1}_{G_{M}^{\varepsilon}(T)} \int_{0}^{T} \int_{s}^{\psi_{n}(s)}\left\|u^{0}(r)\right\|^{2} d r d s\right) \\
& \leq 2 \eta C 2^{-n} M+2 C_{\eta} \cdot a_{0}^{2} \cdot M^{2} \mathbb{E}\left(\mathbf{1}_{G_{M}^{\varepsilon}(T)} \int_{0}^{T} d r \int_{\left(r-c 2^{-n}\right) \vee 0}^{r}\left\|u^{0}(r)\right\|^{2} d s\right) \\
& \leq 2 \eta C M 2^{-n}+2 C_{\eta} \cdot a_{0}^{2} \cdot M^{3} 2^{-n} ; \\
& J_{2} \leq 2 \sqrt{\varepsilon} \lambda(\varepsilon) \mathbb{E}\left(\mathbf{1}_{G_{M}^{\varepsilon}(T)} \int_{0}^{T} \int_{s}^{\psi_{n}(s)} \eta\left\|X^{\varepsilon}(r)\right\|^{2}+C_{\eta}\left|X^{\varepsilon}(r)\right|^{2}\left\|X^{\varepsilon}(s)\right\|_{\mathcal{H}}^{4} d r d s\right) \\
& \leq 2 \sqrt{\varepsilon} \lambda(\varepsilon) \eta C M 2^{-n}+2 \sqrt{\varepsilon} \lambda(\varepsilon) C_{\eta} \cdot M \mathbb{E}\left(\mathbf{1}_{G_{M}^{\varepsilon}(T)} \int_{0}^{T}\left\|X^{\varepsilon}(s)\right\|_{\mathcal{H}}^{4} d s\right) \cdot 2^{-n} \\
& \leq 2 \sqrt{\varepsilon} \lambda(\varepsilon) \eta C M 2^{-n}+2 \sqrt{\varepsilon} \lambda(\varepsilon) C_{\eta} a_{0}^{2} \cdot M \mathbb{E}\left(\mathbf{1}_{G_{M}^{\varepsilon}(T)} \sup _{0 \leq s \leq T}\left|X^{\varepsilon}(s)\right|^{2} \int_{0}^{T}\left\|X^{\varepsilon}(s)\right\|^{2} d s\right) \cdot 2^{-n} \\
& \leq 2 \sqrt{\varepsilon} \lambda(\varepsilon) \eta C M 2^{-n}+2 \sqrt{\varepsilon} \lambda(\varepsilon) C_{\eta} \cdot a_{0}^{2} \cdot M^{3} 2^{-n} ;
\end{aligned}
$$

by (2.4), we have

$$
\begin{aligned}
J_{3} & \leq 2 \mathbb{E}\left(\mathbf{1}_{G_{M}^{\varepsilon}(T)} \int_{0}^{T} \int_{s}^{\psi_{n}(s)} \eta\left\|X^{\varepsilon}(s)\right\|^{2}+C_{\eta}\left\|X^{\varepsilon}(r)\right\|_{\mathcal{H}}^{2}\left\|u^{0}(r)\right\|_{\mathcal{H}}^{2} d r d s\right) \\
& \leq 2 C M 2^{-n}+2 a_{0}^{2} C_{\eta} \mathbb{E}\left(\mathbf{1}_{G_{M}^{\varepsilon}(T)} \int_{0}^{T} \int_{s}^{\psi_{n}(s)}\left|X^{\varepsilon}(r)\right| \cdot\left\|X^{\varepsilon}(r)\right\| \cdot\left|u^{0}(r)\right| \cdot\left\|u^{0}(r)\right\| d r d s\right) \\
& \leq 2 C M 2^{-n}+2 C_{\eta} a_{0}^{2} M \mathbb{E}\left(\mathbf{1}_{G_{M}^{\varepsilon}(T)} \int_{0}^{T} \int_{s}^{\psi_{n}(s)}\left\|X^{\varepsilon}(r)\right\|\left\|u^{0}(r)\right\| d r d s\right) \\
& \leq 2 C M 2^{-n}+C_{\eta} a_{0}^{2} M \mathbb{E}\left(\mathbf{1}_{G_{M}^{\varepsilon}(T)} \int_{0}^{T} \int_{s}^{\psi_{n}(s)}\left\|X^{\varepsilon}(r)\right\|^{2}+\left\|u^{0}(r)\right\|^{2} d r d s\right) \\
& \leq 2 C M 2^{-n}+C_{\eta} a_{0}^{2} M^{2} 2^{-n},
\end{aligned}
$$

here integral transformation as in $J_{1}$ has been applied to obtain the last inequality.

Similarly as $J_{3}$, we have

$$
I_{3} \leq 2 \mathbb{E}\left(\mathbf{1}_{G_{M}^{\varepsilon}(T)} \int_{0}^{T} \int_{s}^{\psi_{n}(s)}\left|\left(B\left(u^{0}(r), X^{\varepsilon}(r)\right), X^{\varepsilon}(s)\right)\right| d r d s\right)
$$




$$
\begin{aligned}
& \leq 2 \mathbb{E}\left(\mathbf{1}_{G_{M}^{\varepsilon}(T)} \int_{0}^{T} \int_{s}^{\psi_{n}(s)} \eta\left\|X^{\varepsilon}(s)\right\|^{2}+C_{\eta}\left\|X^{\varepsilon}(r)\right\|_{\mathcal{H}}^{2}\left\|u^{0}(r)\right\|_{\mathcal{H}}^{2} d r d s\right) \\
& \leq C M 2^{-n}+C_{\eta} a_{0}^{2} M^{2} 2^{-n} .
\end{aligned}
$$

It is easy to see

$$
\begin{aligned}
I_{4} & \leq 2 \mathbb{E}\left(\mathbf{1}_{G_{M}^{\varepsilon}(T)} \int_{0}^{T} \int_{s}^{\psi_{n}(s)}\left|X^{\varepsilon}(r)-X^{\varepsilon}(s)\right| \cdot R_{1}\left|X^{\varepsilon}(r)\right| d r d s\right) \\
& \leq C_{T} M 2^{-n} .
\end{aligned}
$$

Because of $G_{M}^{\varepsilon}(T) \subset G_{M}^{\varepsilon}(r)$, we have

$$
\begin{aligned}
I_{5} & \leq 2 \lambda^{-1}(\varepsilon) \int_{0}^{T} \mathbb{E}\left(\int_{s}^{\psi_{n}(s)} \mathbf{1}_{G_{M}^{\varepsilon}(r)}\left|X^{\varepsilon}(r)-X^{\varepsilon}(s)\right|^{2} \cdot\left|\sigma\left(r, \sqrt{\varepsilon} \lambda(\varepsilon) X^{\varepsilon}(r)+u^{0}(r)\right)\right|_{L_{Q}}^{2} d r\right)^{\frac{1}{2}} d s \\
& \leq 2 \lambda^{-1}(\varepsilon) \int_{0}^{T} \mathbb{E}\left(\int_{s}^{\psi_{n}(s)} \mathbf{1}_{G_{M}^{\varepsilon}(r)}\left|X^{\varepsilon}(r)-X^{\varepsilon}(s)\right|^{2} \cdot\left(K_{0}+K_{1}\left|\sqrt{\varepsilon} \lambda(\varepsilon) X^{\varepsilon}(r)+u^{0}(r)\right|^{2}\right) d r\right)^{\frac{1}{2}} d s \\
& \leq \lambda^{-1}(\varepsilon) C M 2^{-\frac{n}{2}} ; \\
I_{6} & \leq 2 \mathbb{E}\left(\mathbf{1}_{G_{M}^{\varepsilon}(T)} \int_{0}^{T} \int_{s}^{\psi_{n}(s)}\left|X^{\varepsilon}(r)-X^{\varepsilon}(s)\right| \cdot\left|\sigma\left(r, \sqrt{\varepsilon} \lambda(\varepsilon) X^{\varepsilon}(r)+u^{0}(r)\right)\right|_{L_{Q}} \cdot\left|\dot{\phi}^{\varepsilon}(r)\right| d r d s\right) \\
& \leq C M \mathbb{E}\left(\mathbf{1}_{G_{M}^{\varepsilon}(T)} \int_{0}^{T} \int_{s}^{\psi_{n}(s)}\left|\dot{\phi}^{\varepsilon}(r)\right| d r d s\right) \\
& \leq C M \mathbb{E}\left(\mathbf{1}_{G_{M}^{\varepsilon}(T)} \int_{0}^{T}\left(\int_{s}^{\psi_{n}(s)}\left|\dot{\phi}^{\varepsilon}(r)\right|^{2} d r \cdot \int_{s}^{\psi_{n}(s)} d r\right)^{\frac{1}{2}} d s\right) \\
& \leq C_{T} M \cdot N^{\frac{1}{2}} \cdot 2^{-\frac{n}{2}} .
\end{aligned}
$$

Recall (1.4), we have for all sufficient small $\varepsilon$,

$$
\begin{aligned}
I_{7} & \leq \mathbb{E}\left(\mathbf{1}_{G_{M}^{\varepsilon}(T)} \int_{0}^{T} \int_{s}^{\psi_{n}(s)} \frac{1}{\lambda^{2}(\varepsilon)}\left(K_{0}+K_{1}\left|\sqrt{\varepsilon} \lambda(\varepsilon) X^{\varepsilon}(r)+u^{0}(r)\right|^{2}\right) d r d s\right) \\
& \leq C_{T} M \cdot 2^{-n} .
\end{aligned}
$$

Putting all the estimation of $I_{k}$ into (4.19), we obtain (4.18) which completes the proof.

Proof of Propsition 4.5: From Skorokhod representation theorem, there exists $\left(\tilde{\phi}_{\varepsilon}, \tilde{\phi}, \tilde{W}^{\varepsilon}\right)$ such that $\left(\tilde{\phi}_{\varepsilon}, \tilde{W}^{\varepsilon}\right)$ and $\left(\phi^{\varepsilon}, W\right)$ have the same distribution, the distribution of $\tilde{\phi}$ coincides with that of $\phi$ and $\tilde{\phi}_{\varepsilon}$ converges $\tilde{\phi}$ a.s. in the weak topology of $S_{M}$. To lighten notations, we will write $\left(\tilde{\phi}_{\varepsilon}, \tilde{\phi}, \tilde{W}^{\varepsilon}\right)=\left(\phi^{\varepsilon}, \phi, W\right)$. 
Recall (4.3) and (4.4). Let $Z_{\varepsilon}=X^{\varepsilon}-X^{\phi}$, here $X^{\phi}$ is the solution of (4.3) replaced $h$ by $\phi$, then $Z_{\varepsilon}(0)=0$ and

$$
\begin{aligned}
& d Z_{\varepsilon}(t)+A Z_{\varepsilon}(t) d t+B\left(X^{\varepsilon}(t), \sqrt{\varepsilon} \lambda(\varepsilon) X^{\varepsilon}(t)\right) d t+B\left(Z_{\varepsilon}(t), u^{0}(t)\right) d t+B\left(u^{0}(t), Z_{\varepsilon}(t)\right) d t \\
& +\left((\sqrt{\varepsilon} \lambda(\varepsilon))^{-1}\left[\tilde{R}\left(t, u^{0}(t)+\sqrt{\varepsilon} \lambda(\varepsilon) X^{\varepsilon}(t)\right)-\tilde{R}\left(t, u^{0}(t)\right)\right]-\tilde{R}^{\prime}\left(t, u^{0}(t)\right) X^{\phi}(t)\right) d t \\
= & \lambda^{-1}(\varepsilon) \sigma\left(t, u^{0}(t)+\sqrt{\varepsilon} \lambda(\varepsilon) X^{\varepsilon}(t)\right) d W(t)+\sigma\left(t, u^{0}(t)+\sqrt{\varepsilon} \lambda(\varepsilon) X^{\varepsilon}(t)\right) \dot{\phi}^{\varepsilon}(t) d t \\
& -\sigma\left(t, u^{0}(t)\right) \dot{\phi}(t) d t .
\end{aligned}
$$

Hence

$$
\begin{aligned}
&\left|Z_{\varepsilon}(t)\right|^{2}+2 \int_{0}^{t}\left\|Z_{\varepsilon}(s)\right\|^{2} d s \\
&=2 \int_{0}^{t} \sqrt{\varepsilon} \lambda(\varepsilon) B\left(X^{\varepsilon}(s), X^{\varepsilon}(s), X^{\phi}(s)\right) d s-2 \int_{0}^{t} B\left(Z_{\varepsilon}(s), u^{0}(s), Z_{\varepsilon}(s)\right) d s \\
& \quad-2 \int_{0}^{t}\left((\sqrt{\varepsilon} \lambda(\varepsilon))^{-1}\left[\tilde{R}\left(s, u^{0}(s)+\sqrt{\varepsilon} \lambda(\varepsilon) X^{\varepsilon}(s)\right)-\tilde{R}\left(s, u^{0}(s)\right)\right]-\tilde{R}^{\prime}\left(s, u^{0}(s)\right) X^{\phi}(s), Z_{\varepsilon}(s)\right) d s \\
& \quad+2 \lambda^{-1}(\varepsilon) \int_{0}^{t}\left(\sigma\left(s, u^{0}(s)+\sqrt{\varepsilon} \lambda(\varepsilon) X^{\varepsilon}(s)\right) d W(s), Z_{\varepsilon}(s)\right) \\
&+2 \int_{0}^{t}\left(\sigma\left(s, u^{0}(s)+\sqrt{\varepsilon} \lambda(\varepsilon) X^{\varepsilon}(s)\right) \cdot \dot{\phi}^{\varepsilon}(s)-\sigma\left(s, u^{0}(s)\right) \cdot \dot{\phi}(s), Z_{\varepsilon}(s)\right) d s \\
&+\lambda^{-2}(\varepsilon) \int_{0}^{t}\left|\sigma\left(s, u^{0}(s)+\sqrt{\varepsilon} \lambda(\varepsilon) X^{\varepsilon}(s)\right)\right|_{L_{Q}}^{2} d s \\
& \leq 2 \sqrt{\varepsilon} \lambda(\varepsilon) \int_{0}^{t} \eta\left\|X^{\varepsilon}(s)\right\|^{2}+C_{\eta}\left|X^{\varepsilon}(s)\right|^{2}\left\|X^{\phi}(s)\right\|_{\mathcal{H}}^{4} d s+2 \int_{0}^{t} \eta\left\|Z_{\varepsilon}(s)\right\|^{2}+C_{\eta}\left|Z^{\varepsilon}(s)\right|^{2}\left\|u^{0}(s)\right\|_{\mathcal{H}}^{4} d s \\
&+2 \int_{0}^{t}\left|(\sqrt{\varepsilon} \lambda(\varepsilon))^{-1}\left[\tilde{R}\left(s, u^{0}(s)+\sqrt{\varepsilon} \lambda(\varepsilon) X^{\varepsilon}(s)\right)-\tilde{R}\left(s, u^{0}(s)\right)-\tilde{R}^{\prime}\left(s, u^{0}(s)\right) \sqrt{\varepsilon} \lambda(\varepsilon) X^{\varepsilon}(s)\right]\right| \\
&+\left|Z_{\varepsilon}(s)\right| d s \\
&+2 \int_{0}^{t}\left|\tilde{R}^{\prime}\left(s, u^{0}(s)\right)\right| \cdot\left|Z_{\varepsilon}(s)\right|^{2} d s+2 \lambda^{-1}(\varepsilon) \int_{0}^{t}\left(\sigma\left(s, u^{0}(s)+\sqrt{\varepsilon} \lambda(\varepsilon) X^{\varepsilon}(s)\right) d W(s), Z_{\varepsilon}(s)\right) \\
&+2 \int_{0}^{t}\left(\sigma\left(s, u^{0}(s)\right)\left(\dot{\phi}^{\varepsilon}(s)-\dot{\phi}(s)\right), Z_{\varepsilon}(s)\right) d s+2 \int_{0}^{t} \sqrt{L_{1}} \sqrt{\varepsilon} \lambda(\varepsilon)\left|X^{\varepsilon}(s)\right| \cdot\left|\dot{\phi}^{\varepsilon}(s)\right| \cdot\left|Z_{\varepsilon}(s)\right| d s \\
&+\lambda^{-2}(\varepsilon) \int_{0}^{t} K_{0}+K_{1}\left|u^{0}(s)+\sqrt{\varepsilon} \lambda(\varepsilon) X^{\varepsilon}(s)\right|^{2} d s \\
&:= \sum_{k=1}^{8} I_{k}(t) .
\end{aligned}
$$

Since,

$$
I_{7} \leq \varepsilon \lambda^{2}(\varepsilon) \int_{0}^{t} L_{1}\left|X^{\varepsilon}(s)\right|^{2}\left|\dot{\phi}^{\varepsilon}(s)\right|^{2} d s+\int_{0}^{t}\left|Z_{\varepsilon}(s)\right|^{2} d s:=I_{7}^{\prime}+\int_{0}^{t}\left|Z_{\varepsilon}(s)\right|^{2} d s,
$$


and

$$
\begin{aligned}
I_{3} & =2 \int_{0}^{t}\left|\int_{0}^{1}\left[\tilde{R}^{\prime}\left(s, u^{0}(s)+x \sqrt{\varepsilon} \lambda(\varepsilon) X^{\varepsilon}(s)\right)-\tilde{R}^{\prime}\left(s, u^{0}(s)\right)\right] X^{\varepsilon}(s) d x\right|\left|Z_{\varepsilon}(s)\right| d s \\
& \leq C \sqrt{\varepsilon} \lambda(\varepsilon) \int_{0}^{t}\left|X^{\varepsilon}(s)\right|^{2}\left|Z_{\varepsilon}(s)\right| d s \\
& \leq C \varepsilon \lambda^{2}(\varepsilon) \int_{0}^{t}\left|X^{\varepsilon}(s)\right|^{4} d s+\int_{0}^{t}\left|Z_{\varepsilon}(s)\right|^{2} d s \\
& :=I_{3}^{\prime}+\int_{0}^{t}\left|Z_{\varepsilon}(s)\right|^{2} d s
\end{aligned}
$$

Then,

$$
\begin{aligned}
& \left|Z_{\varepsilon}(t)\right|^{2}+2(1-\eta) \int_{0}^{t}\left\|Z_{\varepsilon}(s)\right\|^{2} d s \\
\leq & I_{1}+I_{2}^{\prime}+I_{3}^{\prime}+I_{5}+I_{6}+I_{7}^{\prime}+I_{8}+\int_{0}^{t}\left(C_{\eta}\left\|u^{0}(s)\right\|_{\mathcal{H}}^{4}+2+2\left|\tilde{R}^{\prime}\left(s, u^{0}(s)\right)\right|\right)\left|Z_{\varepsilon}(s)\right|^{2} d s .
\end{aligned}
$$

Gronwall's inequality implies

$$
\begin{aligned}
& \left|Z_{\varepsilon}(t)\right|^{2}+2(1-\eta) \int_{0}^{t}\left\|Z_{\varepsilon}(s)\right\|^{2} d s \\
\leq & \left(I_{1}+I_{3}^{\prime}+I_{5}+I_{6}+I_{7}^{\prime}+I_{8}\right) \exp \left(\int_{0}^{T} C_{\eta}\left\|u^{0}(s)\right\|_{\mathcal{H}}^{4}+2+2\left(\tilde{R}_{1}^{\prime}+\tilde{R}_{2}^{\prime}\left|u^{0}(s)\right|\right) d s\right) .
\end{aligned}
$$

By Lemma 2.1 and (4.9), we may take $M$ big enough such that $\sup _{h \in S_{N}}\left(\sup _{0 \leq s \leq T}\left|X^{h}(s)\right|^{2}+\int_{0}^{T}\left\|X^{h}(s)\right\|^{2} d s\right) \bigvee\left(\sup _{0 \leq s \leq T}\left|u^{0}(s)\right|^{2}+\int_{0}^{T}\left\|u^{0}(s)\right\|^{2} d s\right) \leq M$. Let $\left.\left.G_{M, \varepsilon}(t)=\left\{\sup _{0 \leq s \leq t}\left|X^{\varepsilon}(s)\right|^{2}\right) \leq M\right\} \cap\left\{\int_{0}^{t}\left\|X^{\varepsilon}(s)\right\|^{2} d s\right) \leq M\right\}$. We split the proof into two steps.

Step 1: $\mathbb{P}\left(\left(G_{M, \varepsilon}(T)\right)^{c}\right) \leq C\left(1+|\xi|^{4}\right) M^{-1}$ which is obtained by Chebysev inequality and (4.8).

Step 2: $\mathbb{E}\left(\mathbf{1}_{G_{M, \varepsilon}(T)}\left(\sup _{0 \leq s \leq T}\left|Z_{\varepsilon}(s)\right|^{2}+\int_{0}^{T}\left\|Z_{\varepsilon}(s)\right\|^{2} d s\right)\right) \rightarrow 0$ as $\varepsilon \rightarrow 0$.

Now we show the Step 2. When $\omega \in G_{M, \varepsilon}(T)$, we have

$$
I_{1} \leq C \sqrt{\varepsilon} \lambda(\varepsilon) \cdot M^{3}, \quad I_{3}^{\prime} \leq C_{T} \epsilon \lambda^{2}(\varepsilon) M^{2}
$$

For $I_{5}$,

$$
\begin{aligned}
\mathbb{E}\left(\mathbf{1}_{G_{M, \varepsilon}(T)} \sup _{0 \leq t \leq T}\left|I_{5}(t)\right|\right) & \leq 2 \lambda^{-1}(\varepsilon) \mathbb{E}\left(\int_{0}^{T} \mathbf{1}_{G_{M, \varepsilon}(t)}\left|\sigma\left(t, u^{0}(t)+\sqrt{\varepsilon} \lambda(\varepsilon) X^{\varepsilon}(t)\right)\right|_{L_{Q}}^{2}\left|Z_{\varepsilon}(t)\right|^{2} d t\right)^{\frac{1}{2}} \\
& \leq C_{T} \lambda^{-1}(\varepsilon) M .
\end{aligned}
$$


Applying Lemma 4.2, similar to (3.23) in 7,

$$
\mathbb{E}\left(\mathbf{1}_{G_{M, \varepsilon}(T)} \sup _{0 \leq t \leq T} I_{6}(t, \varepsilon)\right) \rightarrow 0, \quad \varepsilon \rightarrow 0
$$

And

$$
I_{7}^{\prime} \leq C \varepsilon \lambda^{2}(\varepsilon) \cdot N \cdot M, \quad I_{8} \leq C_{T} \lambda^{-2}(\varepsilon)(1+M) .
$$

Thus, $\lim _{\varepsilon \rightarrow 0} \mathbb{E}\left(\mathbf{1}_{G_{M, \varepsilon}(T)}\left(\sup _{0 \leq s \leq T}\left|Z_{\varepsilon}(s)\right|^{2}+\int_{0}^{T}\left\|Z_{\varepsilon}(s)\right\|^{2} d s\right)\right)=0$. So Step 2 is obtained.

Finally, since for any $\delta>0$,

$$
\begin{aligned}
& \mathbb{P}\left(\sup _{0 \leq s \leq T}\left|Z_{\varepsilon}(s)\right|^{2}+\int_{0}^{T}\left\|Z_{\varepsilon}(s)\right\|^{2} d s>\delta\right) \\
\leq & \mathbb{P}\left(\left(G_{M, \varepsilon}(T)\right)^{c}\right)+1 / \delta \mathbb{E}\left(\mathbf{1}_{G_{M, \varepsilon}(T)}\left(\sup _{0 \leq s \leq T}\left|Z_{\varepsilon}(s)\right|^{2}+\int_{0}^{T}\left\|Z_{\varepsilon}(s)\right\|^{2} d s\right)\right),
\end{aligned}
$$

Step 1 and Step 2 implies that $\lim _{\varepsilon \rightarrow 0} \mathbb{P}\left(\sup _{0 \leq s \leq T}\left|Z_{\varepsilon}(s)\right|^{2}+\int_{0}^{T}\left\|Z_{\varepsilon}(s)\right\|^{2} d s>\delta\right)=0$, which concludes the proof of the proposition.

Proof of Propsition 4.6; , The proof is similar as that of Proposition 4.5 and easier. The proof will be omitted.

\section{Acknowledgment}

This work was supported by National Natural Science Foundation of China (NSFC) (No. 11431014, No. 11401557), and the Fundamental Research Funds for the Central Universities (No. 0010000048).

\section{References}

1. U. Baier and M.I. Freidlin, Theorems on large deviations and stability under random perturbations. Dokl. Akad. Nauk USSR 235(1977) 253-256.

2. A. Budhiraja and P. Dupuis, A variational representation for positive functionals of infinite dimensional Brownian motion. Probab. Math. Statist. 20(2000) 39-61.

3. A. Budhiraja, P. Dupuis and A. Ganguly, Moderate Deviation Principle for Stochastic Differential Equations with Jump, arXiv:1401.73v1.

4. X. Chen, Probabilities of moderate deviations for B-valued independent random vectors. Chinese J. Contemporary Math. 11(1990) 381-393.

5. X. Chen, Probabilities of moderate deviations for independent random vectors in a Banach space. Chinese J. Appl. Probab. Statist. 7(1991) 24-33.

6. X. Chen, On lower bound of the moderate deviations of i.i.d. random variables in Banach space. Chinese J. Appl. Probab. Statist. 9(1993) 386-393.

7. I. Chueshov and A. Millet, Stochastic 2D Hydrodynamics Type Systems: Well Posedness and Large Deviations. Appl. Math. Optim. 61(2010) 379-420.

8. A. Dembo and O. Zeitouni, Large deviations techniques and applications, (2000) Springer-Verlag, New York. 
9. H. Djellout and A. Guillin, Moderate deviations for Markov chains with atom Stochastic processes and their applications. Stoch. Proc. Appl. 95(2)(2001) 203-221.

10. Z. Dong, J. Xiong, J.L. Zhai and T.S. Zhang, A Moderate Deviation Principle for 2-D Stochastic Navier-Stokes Equations Driven by Multiplicative Lévy noises, arXiv:1505.04671.

11. R. Douc, A. Guillin and J. Najim, Moderate Deviations for Particle Filtering. Ann. Appl. Probab. 15(1B)(2001) 587-614.

12. P. Del Moral, S.L. Hu and L.M. Wu, Moderate Deviations for Mean Field Particle Models arXiv:1204.3308.

13. A. Guillin, Averaging principle of SDE with small diffusion: moderate deviations. Ann. Probab. 31(2003) 413-443.

14. F.Q. Gao, Moderate deviations for martingales and mixing random processes. Stoch. Proc. Appl. 61(1996) 263-275.

15. F.G. Gao, Moderate deviations and large deviations for kernel density estimators. J. Theor. Probab. 16(2003) 401-418.

16. M. Ledoux, Sur les déviations modérées des sommes de variables aléatoires vectorielles indépendants de même loi. Ann. Inst. Henri Poincaré Probab. Statist. 28(1992) 267280.

17. R. Wang and T.S. Zhang, Moderated deviations for stochastic reaction-diffusion equations with multiplicative noise. Potential Anal. 42(1)(2015) 99C-113.

18. R. Wang and J.L. Zhai, A central limit theorem and moderate deviations for 2-D Stochastic Navier-Stokes equations with jumps. arXiv:1505.03021.

19. R. Wang, J.L. Zhai, and T.S. Zhang, A moderate deviation principle for 2-D stochastic Navier-Stokes equations. J. Differential Equations 258(10)(2015) 3363-C3390.

20. L.M. Wu, Moderate deviations of dependent random variables related to CLT. Ann. Probab. 23(1) (1995) 420-445.

21. L.M. Wu, Forward-Backward martingale decomposition and compactness results for additive functionals of stationnary ergodic Markov processes. Annales de l'institut Henri Poincar (B) Probabilits et Statistiques section B 35(2)(1999) 121-141. 